"This is the peer reviewed version of the following article: Eraso-Pichot A, BrasóVives M, Golbano A, Menacho C, Claro E, Galea E, Masgrau R. GSEA of mouse and human mitochondriomes reveals fatty acid oxidation in astrocytes. Glia. 2018 Aug;66(8):1724-1735, which has been published in final form at https://onlinelibrary.wiley.com/doi/10.1002/glia.23330. This article may be used for non-commercial purposes in accordance with Wiley Terms and Conditions for Use of Self-Archived Versions. This article may not be enhanced, enriched or otherwise transformed into a derivative work, without express permission from Wiley or by statutory rights under applicable legislation. Copyright notices must not be removed, obscured or modified. The article must be linked to Wiley's version of record on Wiley Online Library and any embedding, framing or otherwise making available the article or pages thereof by third parties from platforms, services and websites other than Wiley Online Library must be prohibited."

\title{
GSEA of mouse and human mitochondriomes reveals fatty acid oxidation in astrocytes
}

\author{
Abel Eraso-Pichot ${ }^{1 *}$., Marina Brasó-Vives ${ }^{2 *}$., Arantxa Golbano ${ }^{1}$., Carmen Menacho ${ }^{1}$., Enrique \\ Claro $^{1}$, Elena Galea ${ }^{1,3 \#}$ and Roser Masgrau ${ }^{1 \#}$ \\ ${ }^{1}$ Institut de Neurociències i Unitat de Bioquímica de Medicina, Departament de Bioquímica i Biologia \\ Molecular, Universitat Autònoma de Barcelona, 08193 Barcelona, Spain \\ ${ }^{2}$ Institute of Evolutionary Biology (Universitat Pompeu Fabra - CSIC), PRBB, 08003 Barcelona, Spain \\ ${ }^{3}$ ICREA, Passeig Lluís Companys 23, 08010 Barcelona, Spain \\ * A.E-P and M.B-V contributed equally to this work \\ \# To whom correspondence may be addressed. Email: roser.masgrau@uab.cat or elena.galea@uab.cat
}

Running Title: Astrocytes oxidize fatty acids

\section{Main points:}

- Fatty acid oxidation genes are highly expressed in human and mouse astrocytes.

- Fatty acid oxidation genes are upregulated upon maturation and downregulated in injury.

- Fatty acids are oxidized in the presence of glucose but not glutamate.

\section{Abstract}

The prevalent view in neuroenergetics is that glucose is the main brain fuel, with neurons being mostly oxidative and astrocytes glycolytic. Evidence supporting that astrocyte mitochondria are functional has been overlooked. Here we sought to determine what is unique about astrocyte mitochondria by performing unbiased statistical comparisons of the mitochondriome in astrocytes and neurons. Using MitoCarta, a compendium of mitochondrial proteins, together with transcriptomes of mouse neurons and astrocytes, we generated cellspecific databases of nuclear genes encoding for mitochondrion proteins, ranked according to relative expression. Standard and in-house Gene Set Enrichment Analyses (GSEA) of five mouse transcriptomes revealed that genes encoding for enzymes involved in fatty acid oxidation (FAO) and amino acid catabolism are consistently more expressed in astrocytes than in 
neurons. FAO and oxidative-metabolism-related genes are also up-regulated in human cortical astrocytes versus the whole cortex, and in adult astrocytes versus fetal astrocytes. We thus present the first evidence of FAO in human astrocytes. Further, as shown in vitro, FAO coexists with glycolysis in astrocytes and is inhibited by glutamate. Altogether, these analyses provide arguments against the glucose-centered view of energy metabolism in astrocytes and reveal mitochondria as specialized organelles in these cells.

\section{INTRODUCTION}

Brain activity requires essential and complex metabolic interactions between neurons and astrocytes. Examples of such crosstalk are the Glutamate-Glutamine Brain Cycle (GGBC) and the Astrocyte-Neuron Lactate Shuttle (ANLS) hypothesis (Belanger, Allaman, \& Magistretti, 2011). The GGBC frames astrocytes as key regulators of extracellular glutamate clearance. Glutamate released from synapses is transported into astrocytes and transformed to glutamine by glutamine synthetase. Glutamine is then delivered to neurons and converted again to glutamate by the mitochondrial enzyme glutaminase. The ANLS hypothesis proposes that astrocytes produce lactate from glucose by aerobic glycolysis (in the presence of oxygen). Lactate is then transferred to neurons via monocarboxylate transporters. In neurons, lactate is first converted to pyruvate by lactate dehydrogenase, then to acetyl-CoA by pyruvate dehydrogenase, and further oxidized in the Tricarboxylic Acid Cycle (TCA). The coupling of the TCA to the mitochondrial electron transport chain (ETC) would enable neurons to produce ATP. Interestingly, the conversion of lactate into pyruvate in neurons increases the ratio $\mathrm{NADH} / \mathrm{NAD}^{+}$, which could turn off neuronal glycolysis and facilitate the pentose phosphate pathway for synthesis of glutathione and NADP, without sacrificing neuronal energy requirements (Cerdan et al., 2006; Herrero-Mendez et al., 2009). Along these lines, the intrinsic neuronal activity at resting conditions may depend in part on aerobic glycolysis taking place in astrocytes (Raichle, 2015). Lactate availability is necessary to maintain excitatory transmission (Nagase, Takahashi, Watabe, Kubo, \& Kato, 2014), and facilitates synapse formation and growth in development (Goyal, Hawrylycz, Miller, Snyder, \& Raichle, 2014), albeit the source and levels of lactate are controversial (Dienel, 2017). In fact, the ANLS hypothesis has not been proven in humans. Results in favor of or against the hypothesis have been reported from animal studies in vivo (Ashrafi \& Ryan, 2017; Diaz-Garcia et al., 2017; Machler et al., 2016), and illustrate the complexity of brain metabolism.

Whatever the nature and source of the fuels used by neurons may be, the general consensus is that mitochondria are fundamental to neurons, which are oxidative; while astrocytes are glycolytic cells at the service of neuronal energetic needs. This view underestimates the role that mitochondria may play in astrocytes. However, several observations link excitatory neurotransmission with astrocytic anaplerosis and cataplerosis (i.e., reactions that respectively replenish or deplete TCA intermediaries). For example, glutamate taken up by astrocytes is converted in part to alpha-ketoglutarate by oxidative deamination and is incorporated into the TCA cycle (Belanger \& Magistretti, 2009; McKenna, 2007; McKenna et al., 2016; Sonnewald, Westergaard, \& Schousboe, 1997), to generate pyruvate and lactate by cataplerotic reactions (Schousboe, Scafidi, Bak, Waagepetersen, \& McKenna, 2014; Sonnewald, Westergaard, Petersen, Unsgard, \& Schousboe, 1993). Also, astrocytes typically express pyruvate carboxylase. This prototypical anaplerotic enzyme produces oxaloacetate, from which glutamate can be generated if there is an operative TCA 
cycle (Lovatt et al., 2007; Shank, Bennett, Freytag, \& Campbell, 1985; Waagepetersen, Qu, Schousboe, \& Sonnewald, 2001; Yu, Schousboe, \& Hertz, 1982). Moreover, a recent study in isolated mitochondria has revealed comparable oxidative phosphorylation in astrocytes and neurons, despite molecular differences in ETC complexes (Lopez-Fabuel et al., 2016). And, finally, astrocyte mitochondria have an active role in calcium signaling (Agarwal et al., 2017; Eraso-Pichot et al., 2017). This is a key element of astrocyte excitability, mediating astrocyte actions in the so-called cerebrovascular unit that controls the coupling of brain metabolism with blood flow (Belanger et al., 2011).

Overall, the view that astrocytes are only glycolytic is simplistic and underestimates astrocyte metabolic functions. To better understand the complexity of neuroenergetics, we investigated astrocyte mitochondrial functions. On the premise that the functions of mitochondria can be inferred from their molecular makeup, we compared the 'mitochondriome' of astrocytes and neurons. The mitochondriome was based on the MitoCarta (Pagliarini et al., 2008) and on transcriptome databases of cells isolated from four sources: normal mouse brains, mouse spinal cords before and after injury, cultured postnatal astrocytes, and fetal and adult human brains. Functional validation was carried out in cultured astrocytes. The findings indicate that the metabolism of astrocytes is versatile and complex and that mitochondrial fatty acid oxidation (FAO) in astrocytes is a key element in brain metabolism. Our results therefore imply that core tenets in neuroenergetics, such as glucose is the main brain fuel, ought to be revised.

\section{MATERIALS AND METHODS}

2.1. Databases and their treatment. We used MitoCarta (Pagliarini et al., 2008), the mouse transcriptome (Anderson et al., 2016; Cahoy et al., 2008; Doyle et al., 2008; Lovatt et al., 2007; Zhang et al., 2014) and the human brain transcriptome databases (Zhang et al., 2016). We applied the alias2Symbol function from Bioconductor organism packages to convert all gene identifiers from all databases to their official gene symbols. We used logarithm base 2 foldchanges of expression $(\log 2 \mathrm{FC})$ to assess expression differences between conditions. All inhouse $\mathrm{R}$ scripts are available under request. Organelle gene lists were extracted from the "Cellular Component" ontology of the GO terms using AmiGO 2.0 software. Lists of genes used for each organelle are available in Dataset S4.

2.2. Standard Gene Set Enrichment Analysis (GSEA). We performed GSEA with the software provided by the Massachusetts Institute of Technology (Subramanian et al., 2005). Normalized enrichment score (NES) and false discovery rate (FDR) were used to quantify enrichment magnitude and statistical significance, respectively.

2.3. In-house GSEA. We designed an in-house GSEA that uses the proportion of genes with a $\log 2 \mathrm{FC}$ greater than 2 (or lower than -2 ) within a given gene set to determine the magnitude of enrichment under a particular condition or conditions. The statistical significance of the enrichments were assessed post-hoc with an exact binomial test considering the proportion of genes with $\log 2 \mathrm{FC}$ greater than 2 (or lower than -2) with respect to the proportion of overexpressed genes of each cell type in the whole database.

2.4. Tissue culture. Primary cultures of cortical astrocytes were obtained from 1-day-old Sprague-Dawley rats as previously described (Barcelo-Torns et al., 2011). In brief, rats were decapitated and the brain was immediately dissected out, minced and incubated for $10 \mathrm{~min}$ at 
$37^{\circ} \mathrm{C}$ in $\mathrm{Ca}^{2+}$-free Krebs Ringer Buffer (KRB) containing $0.025 \%$ trypsin. Tissue was mechanically triturated and cells were filtered through a 40- $\mu \mathrm{m}$ nylon mesh in the presence of soybean trypsin inhibitor and $170 \mathrm{IU} / \mathrm{ml}$ DNAse. Collected cells were plated in T150 flasks in DMEM medium supplemented with $10 \%$ Fetal Bovine Serum (FBS), 50 units $/ \mathrm{ml}$ penicillin and $50 \mu \mathrm{g} / \mathrm{ml}$ streptomycin. Cells were maintained at $37{ }^{\circ} \mathrm{C}$ in a humidified atmosphere containing $5 \% \mathrm{CO}_{2}$. After reaching confluence, microglia were removed by mechanical shaking and astrocytes were seeded in 24-well plates.

2.5. $\quad \beta$-Oxidation assay. This assay quantifies $\beta$-oxidation measuring $\left[{ }^{3} \mathrm{H}\right] \mathrm{H}_{2} \mathrm{O}$ generated after labeling cells with $\left[{ }^{3} \mathrm{H}\right]$ palmitic acid, as previously described (Cabodevilla et al., 2013). Rat cortical secondary astrocytes were labeled overnight with $1 \mu \mathrm{Ci} / \mathrm{ml}\left[{ }^{3} \mathrm{H}\right]$ palmitic acid (10-30 $\mathrm{nM}$, final concentration) in culture medium containing $0.5 \%$ delipidated bovine serum albumin (BSA). After labeling, cells were washed once with KRB containing BSA and twice with KRB. Cells were then incubated with fresh KRB for the indicated times, at different glucose concentrations. Three hours later, $100 \mu \mathrm{M}$ glutamate was added, if applicable. Etomoxir (30 $\mu \mathrm{M})$, an inhibitor of carnitine palmitoyl transferase 1 and therefore of $\left[{ }^{3} \mathrm{H}\right]$ palmitic acid mitochondrial $\beta$-oxidation, was added to establish the extent of $\left[{ }^{3} \mathrm{H}\right] \mathrm{H}_{2} \mathrm{O}$ production from sources other than mitochondrial $\beta$-oxidation. After the incubations, KRB was transferred to tubes containing $1 \mathrm{ml}$ of a 1:2 slurry of Dowex $1 \times 2$ (chloride form) anionic exchange resin in $\mathrm{H}_{2} \mathrm{O}$. Tubes were vortexed and centrifuged for $2 \mathrm{~min}$ at 1,000 rpm. The resin traps radioactive metabolic intermediates other than $\left[{ }^{3} \mathrm{H}\right] \mathrm{H}_{2} \mathrm{O}$. Finally, $0.1 \mathrm{ml}$ of the supernatants containing $\left[{ }^{3} \mathrm{H}\right] \mathrm{H}_{2} \mathrm{O}$ were counted for radioactivity. All experiments were done in triplicate and included a blank control where $\left[{ }^{3} \mathrm{H}\right] \mathrm{H}_{2} \mathrm{O}$ was determined immediately after the addition of KRB buffer without glucose.

2.6. Reagents: All reagents were from Sigma-Aldrich except $\left[{ }^{3} \mathrm{H}\right]$ palmitic acid which was purchased from Perkin Elmer.

\section{RESULTS}

3.1 Generation of astrocyte and neuron 'mitochondriomes' in healthy adult brains. To identify the functional signature of astrocyte mitochondria with respect to neurons, we used the Mitocarta database - the most comprehensive inventory of the molecular composition of mitochondria, comprising of data obtained from 14 mammalian tissues (Pagliarini et al., 2008). Mitocarta contains 1098 proteins, which were converted into 919 genes with the Bioconductor mouse package (Dataset S1). We first manually classified the mitochondrial components identified by MitoCarta (Dataset S1) into 12 functional categories (inner circle, Figure 1). Each category was further divided into groups. We thus had 12 categories with two levels of subdivision (Figure 1 and Dataset S1). For example, the functional category Cellular energy homeostasis was divided into ATP-related metabolism, Carbohydrate catabolism, Co-factors metabolism, Ketone body metabolism, Lipid catabolism, Lipid synthesis and Oxidative metabolism. The last group, in turn, was subclassified in Anaplerosis and cataplerosis, Oxidative phosphorylation and TCA. Among all the 919 genes analyzed only 69 fell into more than one functional category. Obviously, categories may contain different numbers of genes. Cellular energy homeostasis is the largest group with 287 genes. This already implies which mitochondrial functions could be more important. 
Next, mitochondriomes were generated by extracting nucleus-encoded mitochondrial genes and their relative expressions from the transcriptomes of mouse astrocytes and neurons isolated from whole brains or spinal cords. The brain transcriptomes used are available in five repositories (Anderson et al., 2016; Cahoy et al., 2008; Doyle et al., 2008; Lovatt et al., 2007; Zhang et al., 2014), and in the Glia Network website (http://www.networkglia.eu/en). We will henceforth refer to these databases by the name of the first author of the study, viz. Zhang (Zhang et al., 2014), Doyle (Doyle et al., 2008), Cahoy (Cahoy et al., 2008), Lovatt (Lovatt et al., 2007) and Anderson (Anderson et al., 2016). The studies made use of mice at different ages (Zhang, young animals at 7 postnatal days (P7); Doyle, P17 animals; Cahoy, P1-P30 and Lovatt, 10 to 12-week-old mice). Aldh1/1 BAC (Doyle and Zhang), S100ß-EGFP (Cahoy) or GFAPGFP+/GLT1+ (Lovatt) positive cells were selected as astrocytes, whereas neurons were selected as L1 expressing cells (Zhang), EGFP-negative (Cahoy) and Thy1-YFP-positive (Lovatt). In Doyle, 17 cell types expressing different neuron-specific transgenes under a BAC driven reporter were used. Isolation of cell-specific materials was carried out by a combination of FACS and immunopanning (Zhang and Cahoy), translating ribosome affinity purification (TRAP) (Doyle) and FACS (Lovatt). To determine gene expression, Zhang used RNA-sequencing, whereas the other three studies used the Affymetrix microarray platform. All the mitochondriomes are in Dataset S2. The diversity of cell-selection procedures, isolation protocols and mouse age is a strength of the analysis because it ensures the robustness of the conclusions derived from the comparisons among mitochondriomes.

3.2. Mouse GSEA. The mitochondriomes of astrocytes and neurons were then compared by standard GSEA (Subramanian et al., 2005), which allows detecting statistically significant expression differences of a given set of genes in different phenotypes or conditions. Because the standard GSEA requires raw expression data, and these are only available in the Cahoy, Doyle and Zhang databases, only these three databases were used at this stage. As in all other analyses in the present manuscript, only MitoCarta-based functional groups with 10 or more genes were analyzed. Results clearly show that none of the functional groups are overexpressed in neurons versus astrocytes (Figure 2a). By contrast, the two main groups Lipid catabolism/Fatty acid beta-oxidation and Protein catabolism/Amino acid catabolism, are significantly over-expressed in astrocytes versus neurons in all databases except for the second group in the Doyle database. Interestingly, these functional groups belong or are related, in the case of Amino acid catabolism, to the category of Cellular energy homeostasis, suggesting the importance of mitochondrial metabolic functions in astrocytes.

To include Lovatt data in the analyses, we took the log 2 (fold change) of the data $(\log 2 \mathrm{FC})$, and performed an in-house GSEA. In brief, this method gives us the percentage of over-expressed genes $(\log 2 \mathrm{FC}>2$ or $<-2)$ in each functional group and gene-set percentages in neurons and astrocytes are compared with a binomial analysis. The in-house GSEA showed a high coincidence with the previous standard GSEA. No functional group is over-represented in neurons compared to astrocytes, but three groups are over-represented in astrocytes compared to neurons in at least two transcriptomes: Cellular-energy homeostasis/Lipid catabolism/Fatty acid beta-oxidation, Protein homeostasis/Protein catabolism/Amino acid catabolism, and Amine, alcohol and aldehyde metabolism (Figure 2b). Since the Lovatt database presents slightly different patterns of gene expression, we performed a correlation analysis of gene expression among databases. The Zhang, Doyle and Cahoy databases present 
high correlation coefficients between each other, and low correlations with Lovatt (Figure 3a). One can speculate that, different subpopulations of astrocytes were selected for the Lovatt study, or that older mice brains perform less oxidative metabolism (discussed below).

Overall, the data indicate over-representation of mitochondrial gene expression in astrocytes versus neurons. Importantly, this over-representation is not due to transcriptome bias, because the distribution of genes according to their relative expression in astrocytes versus neurons is even when all genes are considered. It is shifted to astrocytes in all transcriptomes-except Zhang-when only mitochondrial genes are taken into consideration (Figure 3b). Specifically, 131 genes of mitochondrial proteins are over-expressed ( $\log 2 \mathrm{FC}>2$ ) in astrocytes compared to neurons versus 24 genes over-expressed in neurons compared to astrocytes ( $\log 2 \mathrm{FC}<-2)$ in Doyle; 87 versus 20 in Cahoy; 74 versus 25 in Zhang and 50 versus 15 in the Lovatt database. The results contradict the prevailing view that astrocyte mitochondria are of lesser importance than neuronal mitochondria.

3.3. Fatty acid oxidation in astrocytes. To gain further insight into the distinct functional signature of astrocyte mitochondria, we examined the individual genes over-expressed in astrocytes in at least two transcriptomes (Table 1). In Cellular energy homeostasis, 19 out of the 36 over-expressed genes are related to canonical FAO. Notably, they encompass all the enzymes associated with this pathway (Figure 4b), including long-chain fatty acid transport to mitochondria by Ctp1a and Ctp2, in agreement with recent findings (Jernberg, Bowman, Wolfgang, \& Scafidi, 2017). There is also over-expression of enzymes related to the oxidation of unsaturated fatty acids, namely Decr1, Eci1 and Eci2 (Table 1). In addition to FAO genes, the over-expression of three groups of genes in astrocytes versus neurons supports that FAO does occur in astrocyte mitochondria. First, there are Pdk4 and Pdk2, genes that encode for pyruvate dehydrogenase kinases, which inhibit pyruvate dehydrogenase, preventing pyruvate conversion to acetyl-CoA. As a consequence, malonyl-CoA would not be produced from glucose (Foster, 2012) and would not inhibit the entrance of acyl-CoA (activated fatty acids) into the mitochondrion (Panov, Orynbayeva, Vavilin, \& Lyakhovich, 2014). Second, there are genes related to enzymes that, if acetyl-CoA comes from FAO rather than from glucose oxidation, would ensure the replenishment of TCA intermediates through the following reactions: (i) anaplerotic reactions such as the transformation of pyruvate into oxaloacetate by pyruvate carboxylase (PCX); (ii) catabolism of glycogenic amino acids such as Glycine (Gldc, Gcsh), Alanine (Gpt2), Valine (Hibadh, Hibch, Aldh6a1), Serine (Sdsl, Serhl) and Proline (Prodh) in astrocytes; and (iii) conversion of glutamate to alpha-ketoglutarate, as there is high expression of S/c25a18 in astrocytes, which transports glutamate to the mitochondria. Also there is high expression of Glud1, a glutamate dehydrogenase that catalyzes the oxidative deamination of glutamate to alpha-ketoglutarate (McKenna, 2007). Third, there are genes related to protection against oxidative stress. The concern that FAO may cause excessive oxidative stress (Schonfeld \& Reiser, 2017) is unwarranted because there is over-expression of Prdx6 in astrocytes, an enzyme participating in the reduction of $\mathrm{H}_{2} \mathrm{O}_{2}$, and $\mathrm{Msrb2}$, which provides protection against oxidative stress (Ugarte et al., 2013). This agrees with studies showing that astrocytes have higher levels of a variety of antioxidant molecules (including glutathione, ascorbate, and vitamin E) and display greater activities of cytosolic enzymes that detoxify reactive oxygen species such as glutathione S-transferase, glutathione peroxidase, and 
catalase (Belanger et al., 2011). Also there are a further five genes related to Redox homeostasis and aldehyde dehydrogenases over-expressed in astrocytes compared to neurons. Moreover, we should highlight that fatty acid availability is not a limiting step for astrocyte FAO as fatty acids are transported into the brain by either rapid passive diffusion and/or facilitated transport across the Brain Blood Barrier (Ebert, Haller, \& Walton, 2003; Jernberg et al., 2017; Schonfeld \& Reiser, 2013). Further, astrocytes could oxidize fatty acids from intracellular lipid droplets (Cabodevilla et al., 2013; Gubern et al., 2008). All in all, astrocytes appear to be endowed with the necessary molecular tools to carry out mitochondrial FAO.

In this vein, an active TCA cycle fueled by acetyl-CoA from FAO and carbon backbones derived from amino acid catabolism can produce glutamine or pyruvate, from cataplerotic reactions (Lovatt et al., 2007; McKenna, 2007; Sonnewald et al., 1993). Hence glutamine and/or lactate could be supplied to neurons. Neurons could convert glutamine to glutamate via glutaminases $G / s$ and $G / s 2$, which have a higher expression in neurons compared to astrocytes (Table 1). Alternatively, acetyl-CoA from FAO could be diverted to ketogenesis, as astrocytes overexpress Hmgcs 2 which encodes HMG-CoA synthase 2, the rate-limiting enzyme of this pathway (Table 1). But some authors claim ketogenesis in the brain of fed animals is minimal (Ebert et al., 2003).

Altogether, the computerized analyses suggest that FAO is a constitutive metabolic pathway in astrocytes, compatible with glycolysis. To validate this prediction experimentally, we measured FAO in astrocytes by quantifying the production of $\left[{ }^{3} \mathrm{H}\right] \mathrm{H}_{2} \mathrm{O}$ from $\left[{ }^{3} \mathrm{H}\right]$ palmitic acid. We chose cultured astrocytes to demonstrate unequivocally that astrocytes perform FAO. First, we confirmed that cultured astrocytes were a valid model by comparing the mitochondriomes of cultured astrocytes versus astrocytes isolated from adult mice (Cahoy et al., 2008) with the in-house GSEA (Dataset S3). We observed that there is no significant difference in Fatty acid beta-oxidation between cultured and brain-isolated astrocytes (Figure 4a). Second, we detected FAO in astrocytes. In the presence of $10 \mathrm{mM}$ glucose FAO decreased by approximately $50 \%$, but it was not abolished (Figure 4c). The data thus demonstrate the coexistence and interplay of FAO and glycolysis. Third, we examined FAO in the context of acutely increased glycolysis, as it occurs in vivo during task-elicited excitatory neurotransmission. This entails the release of glutamate that stimulates glucose uptake and glycolysis rate in astrocytes (Pellerin \& Magistretti, 1994). To mimic this scenario, $100 \mu \mathrm{M}$ glutamate was added 3 hours after the initial time of measurement of FAO. We observed that glutamate stops the accumulation of $\left[{ }^{3} \mathrm{H}\right] \mathrm{H}_{2} \mathrm{O}$, suggesting a complete inhibition of FAO (Figure 4c). The effect is noticeable as early as 30 minutes after the addition of glutamate, and does not depend on increased glycolysis, because it is observed also in the absence of external glucose (Figure 4c). The data support the capacity of astrocytes to simultaneously use glucose and fatty acids, except under conditions of high excitatory neuronal activity.

3.3. Astrocyte mitochondria upon injury. The data thus far indicate that FAO is constitutive in normal astrocytes and finely regulated. In order to study what happens in injury, we compared with standard GSEA the mitochondriomes of spinal-cord astrocytes versus nonastrocytic cells 2 weeks after spinal-cord injury caused by severe crush and lateral 
compression, a model characterized by the appearance of reactive astrocytes (Anderson et al., 2016). Note that under normal conditions, the expression of mitochondrial genes in spinal cord astrocytes compared to other cell types is similar to astrocytes versus neurons in the healthy forebrain (Figure S1 and Dataset S3). Strikingly, the analysis revealed that mitochondrial functions were globally reduced in astrocytes after injury, with a statistically significant decrease in: Oxidative metabolism/Oxidative phosphorylation and Oxidative metabolism/TCA, Cellular energy homeostasis/Lipid Catabolism/Fatty acid oxidation, Mitochondrial DNA translation, Amino acid catabolism, and Small-molecule transport (Figure 5a). Although, we cannot assign these changes of the mitochondriome to a specific astrocyte population among those reported to appear in the injured brain (Wanner et al., 2013), our data support that fatty acid and amino acid based oxidative metabolism is a normal function of mitochondria that is impaired in brain injury.

3.4. Astrocyte mitochondria in human brains. At present there is no evidence that FAO takes place in the human brain, probably due to the difficulty of measuring such activity in living subjects or in postmortem samples. Therefore, we took advantage of human brain transcriptomes available in Zhang et al., 2016 as a unique resource to elucidate the existence of FAO in human astrocytes. However, we did not perform GSEA-based comparisons between mitochondriomes of human astrocytes and neurons because the neuronal databases are available from only one subject. Instead, we performed standard GSEA comparing astrocyte transcriptomes with transcriptomes from the whole cortex from the same study. The lists of genes used can be found in Dataset S4 and the heat map in Figure 5b. Lipid catabolism/Fatty acid oxidation together with Oxidative metabolism/oxidative phosphorylation and TCA genes, Protein homeostasis and Nucleic acid metabolism/mitochondrial DNA translation are significantly over-expressed in astrocytes compared to whole cortex. The data therefore supports the existence of FAO and oxidative metabolism in human astrocytes.

Mitochondrion activity is associated with cell differentiation (Wanet, Arnould, Najimi, \& Renard, 2015). The databases used so far derive from cells isolated from mice and humans at different ages, suggesting that FAO is present in both young and adult brains. But does it increase or decrease with astrocyte maturation? That is, is mitochondrial activity also crucial for astrocyte maturation? As noted, the comparison between cultured postnatal astrocytes and astrocytes isolated from adult brain serves to argue in favor of a potentiation of astrocyte mitochondrial functions with maturation. To further analyze this hypothesis, we compared, with standard GSEA, mitochondriomes from astrocytes isolated from fetal and adult human brains (Zhang et al., 2016). The lists of genes used can be found in Dataset S4. Almost all mitochondrial functions are over-expressed in adult versus fetal human astrocytes (Figure $5 \mathrm{c}$ ). Specifically, Lipid catabolism/Fatty acid oxidation is, again, overexpressed in a statistically significant manner together with Oxidative metabolism/Oxidative phosphorylation and TCA, Protein catabolism/Amino acid catabolism, and Amine, alcohol and aldehyde metabolism as well as Membrane lipid metabolism and dynamics, Neurotransmitter metabolism and Oxidative stress. All in all, the GSEA suggest that mitochondrial functions are increased in astrocytes during development, and that oxidative metabolism fueled by FAO and amino acid catabolism takes place in adult human astrocytes. 
Finally, we wanted to check if the increased expression of genes encoding for mitochondrial proteins upon maturation is exclusive to this organelle or it is a general trait. To this purpose we examined the distribution of genes encoding for components of different organelles with respect to their relative gene expression in adult versus fetal human astrocytes (in $\log 2 \mathrm{FC}$ ) (Dataset S5). The distribution of 'total genes', genes related to the 'endoplasmic reticulum' and genes related to the 'Golgi apparatus' is even between fetal and adult cells. By contrast, genes encoding for 'nuclear proteins' are over-represented in fetal astrocytes, whereas genes encoding for 'mitochondrial proteins' are over-represented in adult astrocytes (Figure $5 \mathrm{~d}$ ). This indicates that the increased production of mitochondrial constituents during development is a unique feature of this organelle, at least in human astrocytes.

\section{DISCUSSION}

This study represents the most extensive and integrated analysis of the expression of nuclear-genes encoding for mitochondrial proteins in the nervous system, to date. We have used data from: a) four databases generated from mouse brains at different ages using different methodologies either to identify and isolate cells or to analyze gene expression; $b$ ) cultured astrocytes; c) fetal and adult human astrocytes, and d) spinal cord astrocytes. The results support the relevance of astrocyte mitochondria and define different mitochondrial functional signatures in astrocytes versus neurons. Importantly, we strongly suggest that FAO is active in human astrocytes.

Our analyses show that the expression of TCA and oxidative-phosphorylation-related genes in mice is not different in astrocytes and neurons. Previous studies have reached the same conclusions as our analyses. In fact, Lovatt and colleagues reported that genes related to oxidative metabolism are upregulated in astrocytes versus neurons (Lovatt et al., 2007), but our analyses are more restrictive and do not reach statistical significance. Moreover, activities of complexes I-IV of the mitochondrial ETC have been recently shown to be the same in neurons and astrocytes isolated from mouse brains, despite the fact that complex $I$ is predominantly assembled into super complexes in neurons but not in astrocytes (Lopez-Fabuel et al., 2016). That astrocytes are oxidative in humans is also supported by nuclear magnetic resonance in vivo showing mitochondrial glutamate oxidative metabolism after the addition of acetate (Bluml, Moreno-Torres, Shic, Nguy, \& Ross, 2002; Lebon et al., 2002). Despite this evidence, the possibility that astrocytes are oxidative has been overlooked. Indeed, most reviews about brain metabolism, even recent ones, consider glucose as the only energy substrate, FAO being ignored (Belanger et al., 2011; Lopez-Fabuel et al., 2016; Raichle, 2015), or denied (Schonfeld \& Reiser, 2013, 2017). However, FAO was calculated to account for $15-$ $20 \%$ of total brain oxidative energy production (Ebert et al., 2003; Panov et al., 2014), and direct and indirect evidence argue in its favor (Cabodevilla et al., 2013; Ebert et al., 2003; Edmond, Robbins, Bergstrom, Cole, \& de Vellis, 1987; Jernberg et al., 2017; Kawamura, 1988; Panov et al., 2014; Sayre et al., 2017). Nevertheless, which cells perform FAO in whole brains has not been clarified. Our study provides molecular evidence to support that FAO takes place in the CNS, and its compartmentalization is mainly in astrocytes. Unlike previous findings, our conclusions come from an extensive range of transcriptomes of isolated astrocytes, FAO being 
a significant hit in all the astrocyte mitochondriomes analyzed. Robustness of the conclusions is also supported by the fact that all the FAO enzymes are overexpressed in astrocytes versus neurons at least in two mice transcriptomes, and this coincides with human data. A drawback of our study is that it is mainly based on data from transcriptomic studies, and hence it may not precisely reflect metabolic fluxes (Chubukov et al., 2013). Nevertheless transcriptomic studies have previously led to the identification of metabolic pathways (Corominas et al., 2013; $\mathrm{Hu}, \mathrm{Gu}, \mathrm{Sun}, \mathrm{Bai}, \&$ Wang, 2016) and they are a sound approach to address brain metabolism in humans (Barros et al., 2017). Moreover, genomic-based models have been extensively used to study metabolic networks (Duarte et al., 2007) and to simulate metabolic-based phenotypes of cancer cells (Gruetter, Seaquist, \& Ugurbil, 2001; Lewis \& Abdel-Haleem, 2013; Lewis et al., 2010).

Our experiments in vitro confirm the co-existence of aerobic glycolysis and FAO in astrocytes, as forecast in a review (Panov et al., 2014). They also agree with recent findings that suggest FAO occurs in the presence of glucose in mouse hippocampus and cortex (Jernberg et al., 2017). We show that FAO in astrocytes is versatile, because it is inhibited by high concentrations of glutamate, resembling states of high excitatory neuronal activity. It is well established that glutamate up-regulates glycolysis in astrocytes (Bittner et al., 2011; Loaiza, Porras, \& Barros, 2003; Pellerin \& Magistretti, 1994), which may account for [18F]Fluorodeoxyglucose PET neuroimaging (Zimmer et al., 2017). However, the inhibition of FAO by glutamate does not appear to depend on glycolysis, because it also takes place in the absence of glucose. Without ruling out the possible involvement of glutamatergic receptors, glutamate induced inhibition of FAO might be a result of its uptake leading to: 1) an inhibition of the mitochondrial long-chain fatty acid transporter CPT1, as has been shown in permeabilized hepatocytes (Guzman, Velasco, Castro, \& Zammit, 1994); 2) activation of acetylCoA carboxylase, increasing levels of malonyl-CoA, and hence inhibiting CPT1, as shown previously in other tissues (Boone, Chan, Kulpa, \& Brownsey, 2000); and/or 3) acidification of mitochondria and reduction of oxygen consumption (Azarias et al., 2011). Whatever the precise mechanism of FAO inhibition might be, it seems paradoxical that glutamate can inhibit FAO while entering the TCA and giving rise to lactate to be delivered to neurons (McKenna, 2007; Sonnewald et al., 1993). However, acetyl-CoA entering the TCA could come from other sources than FAO, namely amino acid catabolism, glucose or glycogen, as previously reported (Dienel \& Cruz, 2004; Nissen et al., 2017; Nissen, Pajecka, Stridh, Skytt, \& Waagepetersen, 2015; Swanson, 1992).

We suggest that FAO is key for brain metabolism. The fine regulated use of glucose, fatty acids and amino acids by astrocytes provide the brain with high flexibility. FAO also ensures ATP production needed under different physiological or pathological conditions. In this regard, our observations that FAO enzymes are downregulated in spinal cord injury agree with a metabolic depression suggested after acute spinal cord trauma (Levine et al., 2016) and with observations that FAO is protective in stroke (Sayre et al., 2017). Thus we propose a role of FAO in brain disease progression and that its restoration could have a therapeutic benefit. Considering that astrocytes become a heterogeneous population after spinal cord injury including reactive and newly generated astrocytes (Wanner et al., 2013), future studies should 
clarify the fate of FAO in each astrocyte type at different times after injury, and what happens to astrocyte $F A O$ in neurodegenerative diseases.

A most important implication of the existence of a versatile mitochondrial metabolism in astrocytes of adult human brain, including oxidative metabolism by way of FAO and amino acid catabolism, is the interpretation of imaging techniques that monitor neuronal activity based on oxygen consumption or availability. An example is BOLD-MRI, which is widely used to monitor neuronal activity, and that also depends on blood flow. First, if astrocyte FAO consumes high amounts of $\mathrm{O}_{2}$ and it is inhibited by glutamate, it follows that the increase in glucose consumption in astrocytes triggered by excitatory neuronal activity would be accompanied by a decrease in $\mathrm{O}_{2}$ consumption. This might explain one of the big enigmas in neuroenergetics and neuroimaging: why are task-elicited increases in blood flow and glucose consumption only marginally matched by increases in $\mathrm{O}_{2}$ consumption (Belanger et al., 2011; Fox, Raichle, Mintun, \& Dence, 1988)? Second, is astrocyte FAO coupled to blood flow and $\mathrm{O}_{2}$ availability, as measured by the BOLD signal? Third, beyond task-dependent metabolic requirements-the most studied paradigm in the relationship between brain function and energy metabolism; does astrocyte FAO sustain astrocyte functions during intrinsic activity, the activity of the brain in the absence of external inputs (Raichle, 2015)?

Finally, we demonstrated the over-expression of genes encoding for mitochondrial proteins compared to other organelles in adult human astrocytes, including FAO, amino acid catabolism and oxidative metabolism-related genes. Our results are an important addition to findings showing that glycogen metabolism, aerobic glycolysis and lactate release are markers of astrocyte differentiation from neural stem cells (Brunet, Allaman, Magistretti, \& Pellerin, 2010; Brunet et al., 2004). Our data confirm that astrocytes also follow the golden rule of increased mitochondrial activity and metabolism during differentiation (Wanet et al., 2015), but reveal cell-specific particularities. For example, when neurogenesis is induced by PGC1alfamediated mitochondrial biogenesis, there is no upregulation or downregulation of FAO enzymes (Stoll et al., 2015). However, according to our data, astrocyte maturation implies an increase in FAO and amino acid catabolism, in agreement with the developmental upregulation of carnitine palmitoyl transferases and acyl-CoA dehydrogenases in young mice (Jernberg et al., 2017). We thus argue that the key point in deriving astrocytes from iPSCs, or neurons from astrocytes, is mitochondrial specialization, and not the switch from a nonoxidative to an oxidative phenotype. Interestingly, a meta-analysis of glucose and oxygen consumption across the human lifespan concluded that anaerobic glycolysis appears to decrease dramatically in the elderly, whereas oxygen consumption is slightly higher (Goyal et al., 2014). This raises the possibility that astrocyte FAO gains importance in aging.

In summary, the study reveals astrocyte mitochondria as key, specialized organelles in the adult brain and broadens the perception that astrocytes are merely glycolytic cells. They are glycolytic and fatty acid and amino acid oxidative cells. This metabolic complexity should be taken into consideration when examining the relationship between brain energy metabolism and function in health and disease, as well as in development.

ACKNOWLEDGMENTS: This research was funded by Grants BFU2012-38844 and BFU201679735-P from MInisterio de Economía y Competitividad, Spanish Government and grant 
ELA2012-033C1B from the European Leukodystrophy Foundation. A.E-P is recipient of the fellowship FPU13/05377 from Ministerio de Educación, Cultura y Deporte, Spanish Government and M.B-V is recipient of the fellowship 2015 FI_B 00730 from Age Spanish ència de Gestió d'Ajuts Universitaris I de Recerca, Generalitat de Catalunya. We thank Professor Pierre Magistretti for critical reading of the manuscript. The authors have no conflict of interest to declare.

\section{REFERENCES}

Agarwal, A., Wu, P. H., Hughes, E. G., Fukaya, M., Tischfield, M. A., Langseth, A. J., . . B Bergles, D. E. (2017). Transient Opening of the Mitochondrial Permeability Transition Pore Induces Microdomain Calcium Transients in Astrocyte Processes. Neuron, 93(3), 587605 e587. doi:10.1016/j.neuron.2016.12.034

Anderson, M. A., Burda, J. E., Ren, Y., Ao, Y., O'Shea, T. M., Kawaguchi, R., . . Sofroniew, M. V. (2016). Astrocyte scar formation aids central nervous system axon regeneration. Nature, 532(7598), 195-200. doi:10.1038/nature17623

Ashrafi, G., \& Ryan, T. A. (2017). Glucose metabolism in nerve terminals. Curr Opin Neurobiol, 45, 156-161. doi:10.1016/j.conb.2017.03.007

Azarias, G., Perreten, H., Lengacher, S., Poburko, D., Demaurex, N., Magistretti, P. J., \& Chatton, J. Y. (2011). Glutamate transport decreases mitochondrial $\mathrm{pH}$ and modulates oxidative metabolism in astrocytes. J Neurosci, 31(10), 3550-3559. doi:10.1523/JNEUROSCI.4378-10.2011

Barcelo-Torns, M., Lewis, A. M., Gubern, A., Barneda, D., Bloor-Young, D., Picatoste, F., . . . Masgrau, R. (2011). NAADP mediates ATP-induced Ca2+ signals in astrocytes. FEBS Lett, 585(14), 2300-2306. doi:10.1016/j.febslet.2011.05.062

Barros, L. F., Bolanos, J. P., Bonvento, G., Bouzier-Sore, A. K., Brown, A., Hirrlinger, J., . . . Weber, B. (2017). Current technical approaches to brain energy metabolism. Glia. doi:10.1002/glia.23248

Belanger, M., Allaman, I., \& Magistretti, P. J. (2011). Brain energy metabolism: focus on astrocyte-neuron metabolic cooperation. Cell Metab, 14(6), 724-738. doi:10.1016/j.cmet.2011.08.016

Belanger, M., \& Magistretti, P. J. (2009). The role of astroglia in neuroprotection. Dialogues Clin Neurosci, 11(3), 281-295.

Bittner, C. X., Valdebenito, R., Ruminot, I., Loaiza, A., Larenas, V., Sotelo-Hitschfeld, T., . . . Barros, L. F. (2011). Fast and reversible stimulation of astrocytic glycolysis by K+ and a delayed and persistent effect of glutamate. J Neurosci, 31(12), 4709-4713. doi:10.1523/JNEUROSCI.5311-10.2011

Bluml, S., Moreno-Torres, A., Shic, F., Nguy, C. H., \& Ross, B. D. (2002). Tricarboxylic acid cycle of glia in the in vivo human brain. NMR Biomed, 15(1), 1-5.

Boone, A. N., Chan, A., Kulpa, J. E., \& Brownsey, R. W. (2000). Bimodal activation of acetyl-CoA carboxylase by glutamate. J Biol Chem, 275(15), 10819-10825.

Brunet, J. F., Allaman, I., Magistretti, P. J., \& Pellerin, L. (2010). Glycogen metabolism as a marker of astrocyte differentiation. J Cereb Blood Flow Metab, 30(1), 51-55. doi:10.1038/jcbfm.2009.207

Brunet, J. F., Grollimund, L., Chatton, J. Y., Lengacher, S., Magistretti, P. J., Villemure, J. G., \& Pellerin, L. (2004). Early acquisition of typical metabolic features upon differentiation of mouse neural stem cells into astrocytes. Glia, 46(1), 8-17. doi:10.1002/glia.10348 
Cabodevilla, A. G., Sanchez-Caballero, L., Nintou, E., Boiadjieva, V. G., Picatoste, F., Gubern, A., \& Claro, E. (2013). Cell survival during complete nutrient deprivation depends on lipid droplet-fueled beta-oxidation of fatty acids. J Biol Chem, 288(39), 27777-27788. doi:10.1074/jbc.M113.466656

Cahoy, J. D., Emery, B., Kaushal, A., Foo, L. C., Zamanian, J. L., Christopherson, K. S., . . B Barres, B. A. (2008). A transcriptome database for astrocytes, neurons, and oligodendrocytes: a new resource for understanding brain development and function. J Neurosci, 28(1), 264-278. doi:10.1523/JNEUROSCI.4178-07.2008

Cerdan, S., Rodrigues, T. B., Sierra, A., Benito, M., Fonseca, L. L., Fonseca, C. P., \& GarciaMartin, M. L. (2006). The redox switch/redox coupling hypothesis. Neurochem Int, 48(6-7), 523-530. doi:10.1016/j.neuint.2005.12.036

Chubukov, V., Uhr, M., Le Chat, L., Kleijn, R. J., Jules, M., Link, H., . . . Sauer, U. (2013). Transcriptional regulation is insufficient to explain substrate-induced flux changes in Bacillus subtilis. Mol Syst Biol, 9, 709. doi:10.1038/msb.2013.66

Corominas, J., Ramayo-Caldas, Y., Puig-Oliveras, A., Estelle, J., Castello, A., Alves, E., . . Folch, J. M. (2013). Analysis of porcine adipose tissue transcriptome reveals differences in de novo fatty acid synthesis in pigs with divergent muscle fatty acid composition. BMC Genomics, 14, 843. doi:10.1186/1471-2164-14-843

Diaz-Garcia, C. M., Mongeon, R., Lahmann, C., Koveal, D., Zucker, H., \& Yellen, G. (2017). Neuronal Stimulation Triggers Neuronal Glycolysis and Not Lactate Uptake. Cell Metab, 26(2), 361-374 e364. doi:10.1016/j.cmet.2017.06.021

Dienel, G. A. (2017). The metabolic trinity, glucose-glycogen-lactate, links astrocytes and neurons in brain energetics, signaling, memory, and gene expression. Neurosci Lett, 637, 18-25. doi:10.1016/j.neulet.2015.02.052

Dienel, G. A., \& Cruz, N. F. (2004). Nutrition during brain activation: does cell-to-cell lactate shuttling contribute significantly to sweet and sour food for thought? Neurochem Int, 45(2-3), 321-351. doi:10.1016/j.neuint.2003.10.011

Doyle, J. P., Dougherty, J. D., Heiman, M., Schmidt, E. F., Stevens, T. R., Ma, G., . . Heintz, N. (2008). Application of a translational profiling approach for the comparative analysis of CNS cell types. Cell, 135(4), 749-762. doi:10.1016/j.cell.2008.10.029

Duarte, N. C., Becker, S. A., Jamshidi, N., Thiele, I., Mo, M. L., Vo, T. D., . . . Palsson, B. O. (2007). Global reconstruction of the human metabolic network based on genomic and bibliomic data. Proc Natl Acad Sci U S A, 104(6), 1777-1782. doi:10.1073/pnas.0610772104

Ebert, D., Haller, R. G., \& Walton, M. E. (2003). Energy contribution of octanoate to intact rat brain metabolism measured by $13 \mathrm{C}$ nuclear magnetic resonance spectroscopy. I Neurosci, 23(13), 5928-5935.

Edmond, J., Robbins, R. A., Bergstrom, J. D., Cole, R. A., \& de Vellis, J. (1987). Capacity for substrate utilization in oxidative metabolism by neurons, astrocytes, and oligodendrocytes from developing brain in primary culture. J Neurosci Res, 18(4), 551561. doi:10.1002/jnr.490180407

Eraso-Pichot, A., Larramona-Arcas, R., Vicario-Orri, E., Villalonga, R., Pardo, L., Galea, E., \& Masgrau, R. (2017). CREB decreases astrocytic excitability by modifying subcellular calcium fluxes via the sigma-1 receptor. Cell Mol Life Sci, 74(5), 937-950. doi:10.1007/s00018-016-2397-5

Foster, D. W. (2012). Malonyl-CoA: the regulator of fatty acid synthesis and oxidation. J Clin Invest, 122(6), 1958-1959.

Fox, P. T., Raichle, M. E., Mintun, M. A., \& Dence, C. (1988). Nonoxidative glucose consumption during focal physiologic neural activity. Science, 241(4864), 462-464.

Goyal, M. S., Hawrylycz, M., Miller, J. A., Snyder, A. Z., \& Raichle, M. E. (2014). Aerobic glycolysis in the human brain is associated with development and neotenous gene expression. Cell Metab, 19(1), 49-57. doi:10.1016/j.cmet.2013.11.020 
Gruetter, R., Seaquist, E. R., \& Ugurbil, K. (2001). A mathematical model of compartmentalized neurotransmitter metabolism in the human brain. Am J Physiol Endocrinol Metab, 281(1), E100-112.

Gubern, A., Casas, J., Barcelo-Torns, M., Barneda, D., de la Rosa, X., Masgrau, R., . . Claro, E. (2008). Group IVA phospholipase A2 is necessary for the biogenesis of lipid droplets. J Biol Chem, 283(41), 27369-27382. doi:10.1074/jbc.M800696200

Guzman, M., Velasco, G., Castro, J., \& Zammit, V. A. (1994). Inhibition of carnitine palmitoyltransferase I by hepatocyte swelling. FEBS Lett, 344(2-3), 239-241.

Herrero-Mendez, A., Almeida, A., Fernandez, E., Maestre, C., Moncada, S., \& Bolanos, J. P. (2009). The bioenergetic and antioxidant status of neurons is controlled by continuous degradation of a key glycolytic enzyme by APC/C-Cdh1. Nat Cell Biol, 11(6), 747-752. doi:10.1038/ncb1881

Hu, G., Gu, W., Sun, P., Bai, Q., \& Wang, B. (2016). Transcriptome Analyses Reveal Lipid Metabolic Process in Liver Related to the Difference of Carcass Fat Content in Rainbow Trout (Oncorhynchus mykiss). Int J Genomics, 2016, 7281585. doi:10.1155/2016/7281585

Jernberg, J. N., Bowman, C. E., Wolfgang, M. J., \& Scafidi, S. (2017). Developmental regulation and localization of carnitine palmitoyltransferases (CPTs) in rat brain. $J$ Neurochem, 142(3), 407-419. doi:10.1111/jnc.14072

Kawamura, N. (1988). Regulation of fatty acid oxidation in rat brain mitochondria: inhibition of high rates of palmitate oxidation by ADP. Arch Biochem Biophys, 264(2), 546-552.

Lebon, V., Petersen, K. F., Cline, G. W., Shen, J., Mason, G. F., Dufour, S., . . Rothman, D. L. (2002). Astroglial contribution to brain energy metabolism in humans revealed by $13 \mathrm{C}$ nuclear magnetic resonance spectroscopy: elucidation of the dominant pathway for neurotransmitter glutamate repletion and measurement of astrocytic oxidative metabolism. J Neurosci, 22(5), 1523-1531.

Levine, J., Kwon, E., Paez, P., Yan, W., Czerwieniec, G., Loo, J. A., . . Wanner, I. B. (2016). Traumatically injured astrocytes release a proteomic signature modulated by STAT3dependent cell survival. Glia, 64(5), 668-694. doi:10.1002/glia.22953

Lewis, N. E., \& Abdel-Haleem, A. M. (2013). The evolution of genome-scale models of cancer metabolism. Front Physiol, 4, 237. doi:10.3389/fphys.2013.00237

Lewis, N. E., Schramm, G., Bordbar, A., Schellenberger, J., Andersen, M. P., Cheng, J. K., . . . Palsson, B. O. (2010). Large-scale in silico modeling of metabolic interactions between cell types in the human brain. Nat Biotechnol, 28(12), 1279-1285. doi:10.1038/nbt.1711

Loaiza, A., Porras, O. H., \& Barros, L. F. (2003). Glutamate triggers rapid glucose transport stimulation in astrocytes as evidenced by real-time confocal microscopy. J Neurosci, 23(19), 7337-7342.

Lopez-Fabuel, I., Le Douce, J., Logan, A., James, A. M., Bonvento, G., Murphy, M. P., . . . Bolanos, J. P. (2016). Complex I assembly into supercomplexes determines differential mitochondrial ROS production in neurons and astrocytes. Proc Natl Acad Sci U S A, 113(46), 13063-13068. doi:10.1073/pnas.1613701113

Lovatt, D., Sonnewald, U., Waagepetersen, H. S., Schousboe, A., He, W., Lin, J. H., . . . Nedergaard, M. (2007). The transcriptome and metabolic gene signature of protoplasmic astrocytes in the adult murine cortex. J Neurosci, 27(45), 12255-12266. doi:10.1523/JNEUROSCI.3404-07.2007

Machler, P., Wyss, M. T., Elsayed, M., Stobart, J., Gutierrez, R., von Faber-Castell, A., . . . Weber, B. (2016). In Vivo Evidence for a Lactate Gradient from Astrocytes to Neurons. Cell Metab, 23(1), 94-102. doi:10.1016/j.cmet.2015.10.010

McKenna, M. C. (2007). The glutamate-glutamine cycle is not stoichiometric: fates of glutamate in brain. J Neurosci Res, 85(15), 3347-3358. doi:10.1002/jnr.21444 
McKenna, M. C., Stridh, M. H., McNair, L. F., Sonnewald, U., Waagepetersen, H. S., \& Schousboe, A. (2016). Glutamate oxidation in astrocytes: Roles of glutamate dehydrogenase and aminotransferases. J Neurosci Res, 94(12), 1561-1571. doi:10.1002/jnr.23908

Nagase, M., Takahashi, Y., Watabe, A. M., Kubo, Y., \& Kato, F. (2014). On-site energy supply at synapses through monocarboxylate transporters maintains excitatory synaptic transmission. J Neurosci, 34(7), 2605-2617. doi:10.1523/JNEUROSCI.4687-12.2014

Nissen, J. D., Lykke, K., Bryk, J., Stridh, M. H., Zaganas, I., Skytt, D. M., . . Waagepetersen, H. S. (2017). Expression of the human isoform of glutamate dehydrogenase, hGDH2, augments TCA cycle capacity and oxidative metabolism of glutamate during glucose deprivation in astrocytes. Glia, 65(3), 474-488. doi:10.1002/glia.23105

Nissen, J. D., Pajecka, K., Stridh, M. H., Skytt, D. M., \& Waagepetersen, H. S. (2015). Dysfunctional TCA-Cycle Metabolism in Glutamate Dehydrogenase Deficient Astrocytes. Glia, 63(12), 2313-2326. doi:10.1002/glia.22895

Pagliarini, D. J., Calvo, S. E., Chang, B., Sheth, S. A., Vafai, S. B., Ong, S. E., . . Mootha, V. K. (2008). A mitochondrial protein compendium elucidates complex I disease biology. Cell, 134(1), 112-123. doi:10.1016/j.cell.2008.06.016

Panov, A., Orynbayeva, Z., Vavilin, V., \& Lyakhovich, V. (2014). Fatty acids in energy metabolism of the central nervous system. Biomed Res Int, 2014, 472459. doi:10.1155/2014/472459

Pellerin, L., \& Magistretti, P. J. (1994). Glutamate uptake into astrocytes stimulates aerobic glycolysis: a mechanism coupling neuronal activity to glucose utilization. Proc Natl Acad Sci U S A, 91(22), 10625-10629.

Raichle, M. E. (2015). The restless brain: how intrinsic activity organizes brain function. Philos Trans R Soc Lond B Biol Sci, 370(1668). doi:10.1098/rstb.2014.0172

Sayre, N. L., Sifuentes, M., Holstein, D., Cheng, S. Y., Zhu, X., \& Lechleiter, J. D. (2017). Stimulation of astrocyte fatty acid oxidation by thyroid hormone is protective against ischemic stroke-induced damage. J Cereb Blood Flow Metab, 37(2), 514-527. doi:10.1177/0271678X16629153

Schonfeld, P., \& Reiser, G. (2013). Why does brain metabolism not favor burning of fatty acids to provide energy? Reflections on disadvantages of the use of free fatty acids as fuel for brain. J Cereb Blood Flow Metab, 33(10), 1493-1499. doi:10.1038/jcbfm.2013.128

Schonfeld, P., \& Reiser, G. (2017). Brain energy metabolism spurns fatty acids as fuel due to their inherent mitotoxicity and potential capacity to unleash neurodegeneration. Neurochem Int. doi:10.1016/j.neuint.2017.03.018

Schousboe, A., Scafidi, S., Bak, L. K., Waagepetersen, H. S., \& McKenna, M. C. (2014). Glutamate metabolism in the brain focusing on astrocytes. Adv Neurobiol, 11, 13-30. doi:10.1007/978-3-319-08894-5_2

Shank, R. P., Bennett, G. S., Freytag, S. O., \& Campbell, G. L. (1985). Pyruvate carboxylase: an astrocyte-specific enzyme implicated in the replenishment of amino acid neurotransmitter pools. Brain Res, 329(1-2), 364-367.

Sonnewald, U., Westergaard, N., Petersen, S. B., Unsgard, G., \& Schousboe, A. (1993). Metabolism of [U-13C]glutamate in astrocytes studied by $13 \mathrm{C}$ NMR spectroscopy: incorporation of more label into lactate than into glutamine demonstrates the importance of the tricarboxylic acid cycle. J Neurochem, 61(3), 1179-1182.

Sonnewald, U., Westergaard, N., \& Schousboe, A. (1997). Glutamate transport and metabolism in astrocytes. Glia, 21(1), 56-63.

Stoll, E. A., Makin, R., Sweet, I. R., Trevelyan, A. J., Miwa, S., Horner, P. J., \& Turnbull, D. M. (2015). Neural Stem Cells in the Adult Subventricular Zone Oxidize Fatty Acids to Produce Energy and Support Neurogenic Activity. Stem Cells, 33(7), 2306-2319. doi:10.1002/stem.2042 
Subramanian, A., Tamayo, P., Mootha, V. K., Mukherjee, S., Ebert, B. L., Gillette, M. A., . . Mesirov, J. P. (2005). Gene set enrichment analysis: a knowledge-based approach for interpreting genome-wide expression profiles. Proc Natl Acad Sci U S A, 102(43), 15545-15550. doi:10.1073/pnas.0506580102

Swanson, R. A. (1992). Physiologic coupling of glial glycogen metabolism to neuronal activity in brain. Can J Physiol Pharmacol, 70 Suppl, S138-144.

Ugarte, N., Ladouce, R., Radjei, S., Gareil, M., Friguet, B., \& Petropoulos, I. (2013). Proteome alteration in oxidative stress-sensitive methionine sulfoxide reductase-silenced HEK293 cells. Free Radic Biol Med, 65, 1023-1036. doi:10.1016/j.freeradbiomed.2013.08.008

Waagepetersen, H. S., Qu, H., Schousboe, A., \& Sonnewald, U. (2001). Elucidation of the quantitative significance of pyruvate carboxylation in cultured cerebellar neurons and astrocytes. J Neurosci Res, 66(5), 763-770. doi:10.1002/jnr.10061

Wanet, A., Arnould, T., Najimi, M., \& Renard, P. (2015). Connecting Mitochondria, Metabolism, and Stem Cell Fate. Stem Cells Dev, 24(17), 1957-1971. doi:10.1089/scd.2015.0117

Wanner, I. B., Anderson, M. A., Song, B., Levine, J., Fernandez, A., Gray-Thompson, Z., . . . Sofroniew, M. V. (2013). Glial scar borders are formed by newly proliferated, elongated astrocytes that interact to corral inflammatory and fibrotic cells via STAT3dependent mechanisms after spinal cord injury. J Neurosci, 33(31), 12870-12886. doi:10.1523/JNEUROSCI.2121-13.2013

Yu, A. C., Schousboe, A., \& Hertz, L. (1982). Metabolic fate of 14C-labeled glutamate in astrocytes in primary cultures. J Neurochem, 39(4), 954-960.

Zhang, Y., Chen, K., Sloan, S. A., Bennett, M. L., Scholze, A. R., O'Keeffe, S., . . Wu, J. Q. (2014). An RNA-sequencing transcriptome and splicing database of glia, neurons, and vascular cells of the cerebral cortex. J Neurosci, 34(36), 11929-11947. doi:10.1523/JNEUROSCI.1860-14.2014

Zhang, Y., Sloan, S. A., Clarke, L. E., Caneda, C., Plaza, C. A., Blumenthal, P. D., . . Barres, B. A. (2016). Purification and Characterization of Progenitor and Mature Human Astrocytes Reveals Transcriptional and Functional Differences with Mouse. Neuron, 89(1), 37-53. doi:10.1016/j.neuron.2015.11.013

Zimmer, E. R., Parent, M. J., Souza, D. G., Leuzy, A., Lecrux, C., Kim, H. I., . . Rosa-Neto, P. (2017). [18F]FDG PET signal is driven by astroglial glutamate transport. Nat Neurosci, 20(3), 393-395. doi:10.1038/nn.4492

\section{Figure Legends}

Figure 1. Functional classification of mitochondrial molecular components identified by MitoCarta. Genes were classified in twelve functional categories each one represented with a different color in the inner circle and labeled on the outer ring. Each functional group was further divided in subcategories ( 2 outer circles).

Figure 2. Heat maps of mitochondrial functions in mouse brain. (a) Standard GSEA analysis of mitochondrial functions (rows) in astrocytes versus neurons according to Zhang, Doyle and Cahoy mouse brain transcriptomes (columns). The color scale indicates Normalized Enrichment Score (NES), from dark blue (over-expressed in neurons) to dark red (overexpressed in astrocytes). Statistical significance is given by the False Discovery Rate (FDR). 
${ }^{*} p<0.01$ and ${ }^{* * *} p<0.0001$. (b) In-house GSEA of astrocyte versus neuronal over-expressed genes in Zhang, Doyle, Cahoy and Lovatt transcriptomes. The color scale represents the percentage of genes over-expressed ( $\log 2 \mathrm{FC}>2$ ) in astrocytes (red) or neurons (blue). ${ }^{*} \mathrm{p}<0.01$, ${ }^{* *} p<0.001$ and ${ }^{* * *} p<0.0001$, according to the exact binomial test.

Figure 3. (a) Correlation analyses between transcriptomes. We plotted the correlation between the different transcriptomes of mice brain: Lovatt, Zhang, Doyle and Cahoy. Each comparison is indicated in the title. Correlation coefficient $\left(R^{2}\right)$ is indicated inside the graph and represents all genes present in each database. (b) Distribution of gene expression of each transcriptome between astrocytes and neurons. Representation of the number of total genes (density) versus the log2FC of gene expression (change of expression of each gene in astrocytes versus neurons). Positive values represent genes more expressed in astrocytes and negative values represent genes higher expressed in the neuronal compartment. The green line accounts for all genes whereas the red line represents those genes present in the MitoCarta database.

Figure 4. Fatty acid oxidation in astrocytes. (a) Heat map of mitochondrial functions in cultured astrocytes. In-house GSEA of over-expressed genes in cultured neonatal cortical and astrocytes acutely isolated from P17 mouse brain. Colors represent percentages of genes over-expressed $(\log 2 \mathrm{FC}>2) .{ }^{*} \mathrm{p}<0.01$ and ${ }^{* * *} \mathrm{p}<0.0001$ by exact binomial test. (b) Schematic representation of genes related to FAO over-expressed in astrocytes in at least two mitochondriomes. (c) FAO assay in cultured cortical astrocytes. Cells were incubated with $\left[{ }^{3} \mathrm{H}\right]$ palmitic acid overnight and $\left[{ }^{3} \mathrm{H}\right] \mathrm{H}_{2} \mathrm{O}$ accumulation, indicative of FAO, was measured incubating the cells with a KrebsRinger buffer with $10 \mathrm{mM}$ glucose or without glucose. Etomoxir (ETX) was used to assess $\left[{ }^{3} \mathrm{H}\right] \mathrm{H}_{2} \mathrm{O}$ accumulation from sources other than mitochondrial $\left[{ }^{3} \mathrm{H}\right]$ palmitic acid oxidation. 100 $\mu \mathrm{M}$ glutamate was added 3 hours after the Krebs-Ringer buffer. Data are mean \pm SD of one representative experiment of 3 .

Figure 5. (a) Heat map of mitochondrial functions after spinal cord injury. Standard GSEA comparing spinal cord astrocytes before and 2 weeks after injury. Colors indicate Normalized Enrichment Score (NES). Groups enriched in WT astrocytes are represented in red while groups enriched in injured astrocytes are represented in green. ${ }^{*} p<0.01,{ }^{* *} p<0.001$ and ${ }^{* * *} p<0.0001$ FDR. (b) Heat map of mitochondrial functions after standard GSEA analysis comparing human astrocytic and whole cortex transcriptomes. The color scale indicates NES, from dark blue (over-expressed in whole cortex) to dark red (over-expressed in astrocytes). ${ }^{*} p<0.01$ and ***p<0.0001 FDR (c) Heat map of mitochondrial functions after standard GSEA analysis comparing adult versus fetal human astrocytes. Color indicates NES, green is enrichment in adult astrocytes and yellow in fetal astrocytes. ${ }^{*} p<0.01,{ }^{* *} p<0.001$ and ${ }^{* * *} p<0.0001$ FDR. (d) Analysis of log2FC distributions of total gene expression for different organelles in adult versus fetal human astrocytes. Positive numbers represent genes highly expressed in adults, while negative numbers represent genes more expressed in fetal stages.

Table 1. List of genes over-expressed in each mitochondrial functional category in astrocytes and neurons in at least two transcriptomes of mice cortex.

Figure S1. Heat map of mitochondrial functions in mouse brain. Standard GSEA of spinal cord astrocytic mitochondrial genes versus 'other cell types'. Colors represent Normalized 
Enrichment Score (NES) of each mitochondrial function over-expressed in astrocytes (red) or 'other cell types' (blue). ${ }^{*} p<0.01,{ }^{* *} p<0.001$ and ${ }^{* * *} p<0.0001$ FDR. 


\section{Amine, alcohol and}

aldehyde metabolism

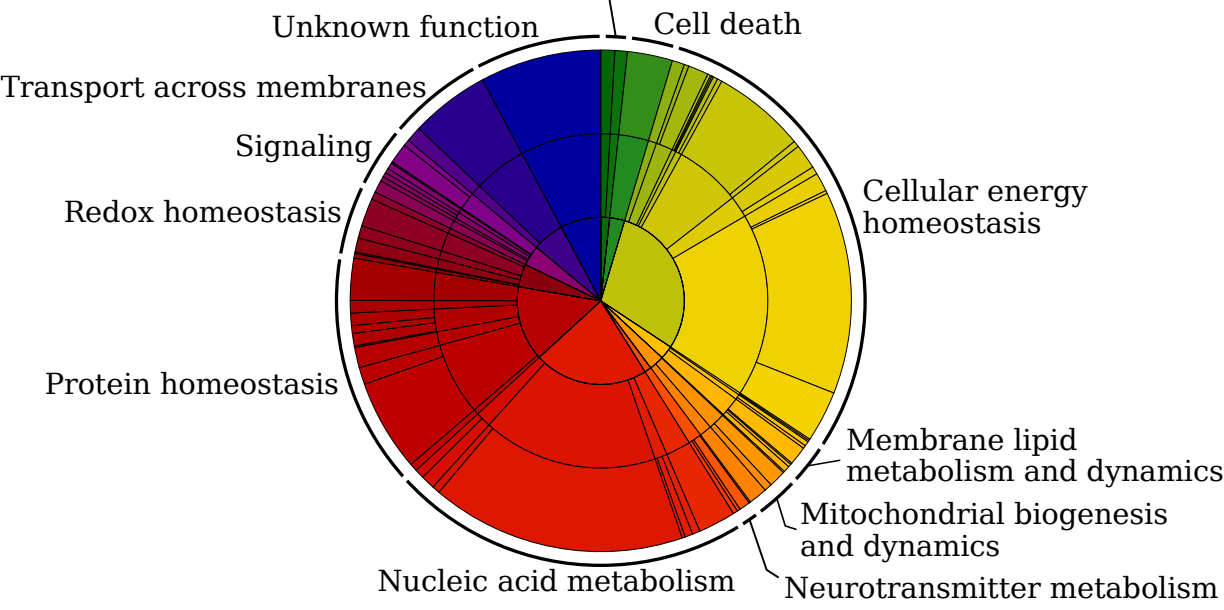


_ Amine, alcohol and aldehyde metabolism

- Cell death

- Cellular energy homeostasis

T Carbohydrate catabolism

- Pyruvate metabolism

T Lipid catabolism

- Fatty acid beta oxidation

T Lipid synthesis

- Fatty acid synthesis

T Oxidative metabolism

- Anaplerosis and cataplerosis

- Oxidative phosphorylation

- TCA

— Membrane lipid metabolism and dynamics Membrane lipid synthesis

_ Glycerophospholipid synthesis

— Mitochondrial biogenesis and dynamics

Mitochondrial morphology

- Mitochondrial trafficking

- Neurotransmitter metabolism

— Nucleic acid metabolism

- Mitochondrial DNA replication

Mitochondrial DNA translation

Nucleotide synthesis

- Protein homeostasis

\section{Protein catabolism}

- Amino acid catabolism

Hydrolysis and metabolism of peptides and proteins

Protein folding

- Protein synthesis

- Transport of proteins

- Redox homeostasis

Oxidative stress

_ Signaling

— Transport across membranes

- Small molecule transport

— Unknown function

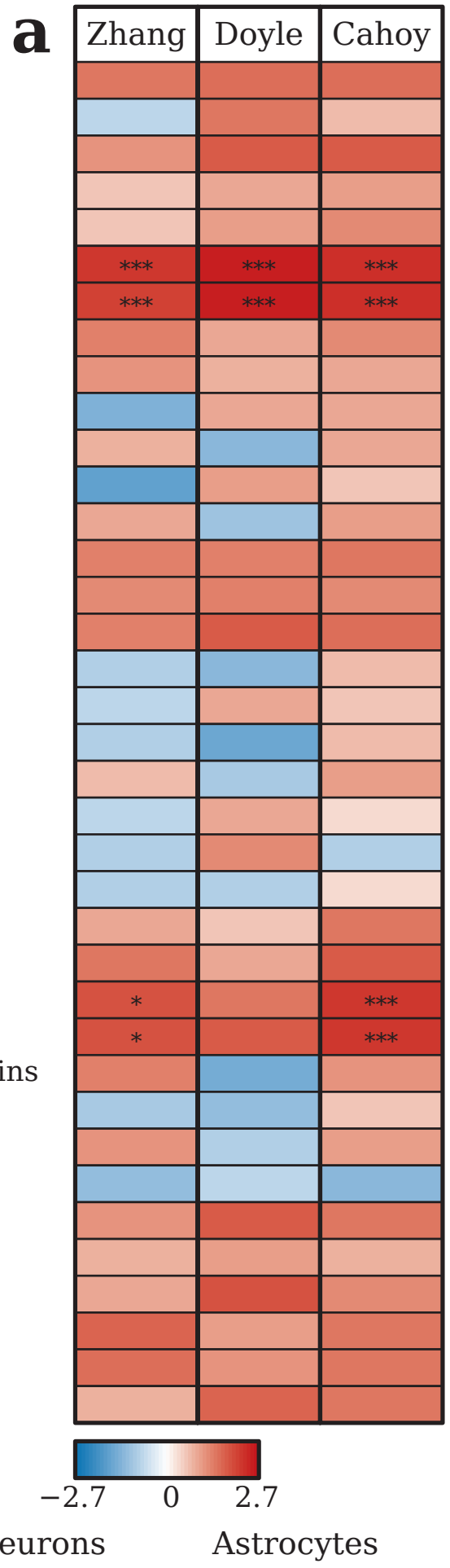

b

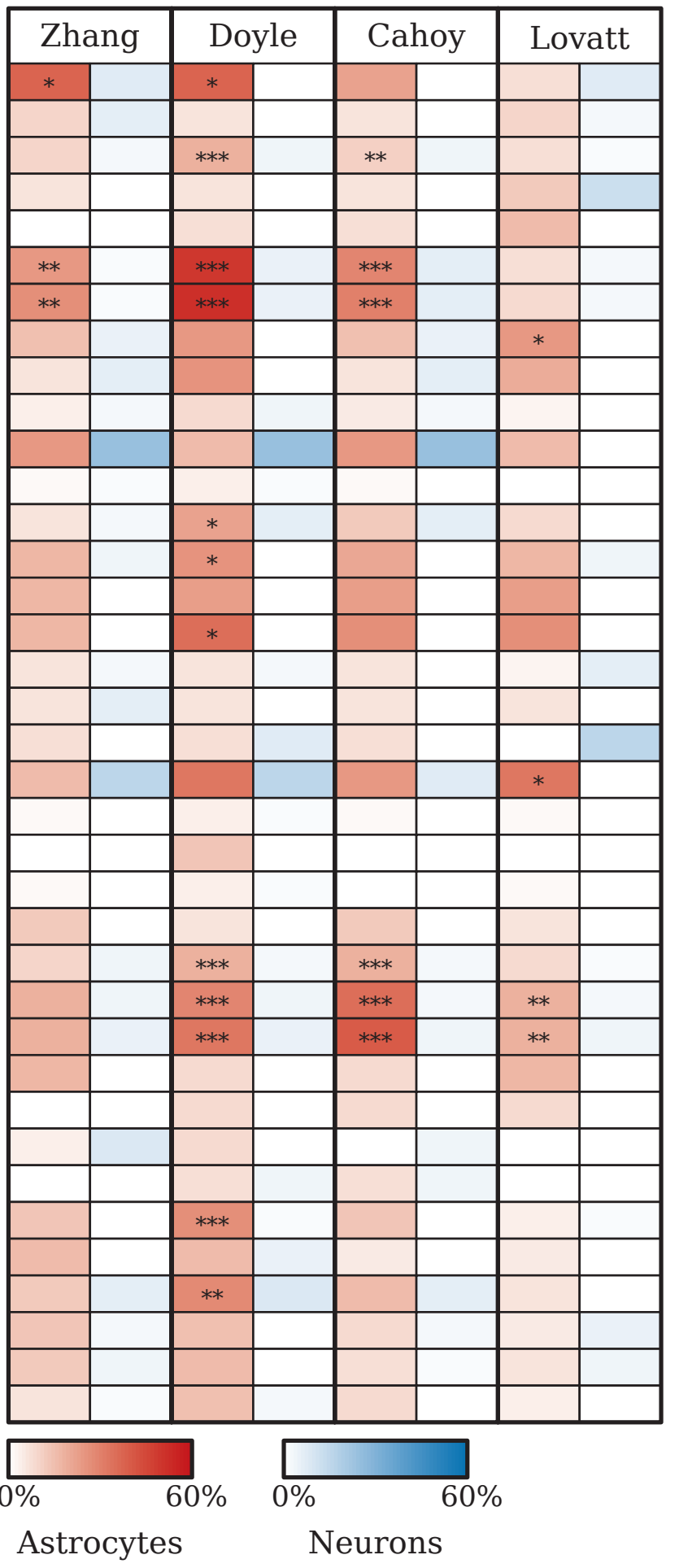




\section{$\mathbf{a}$}
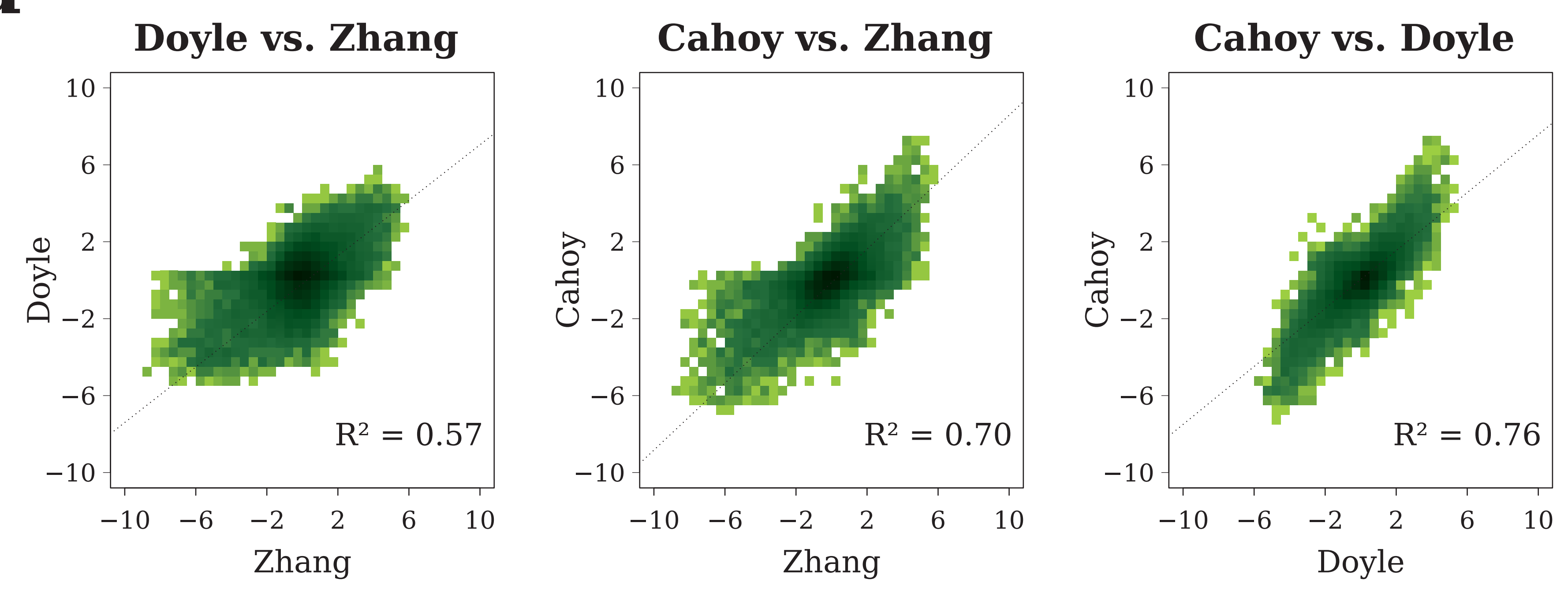

b

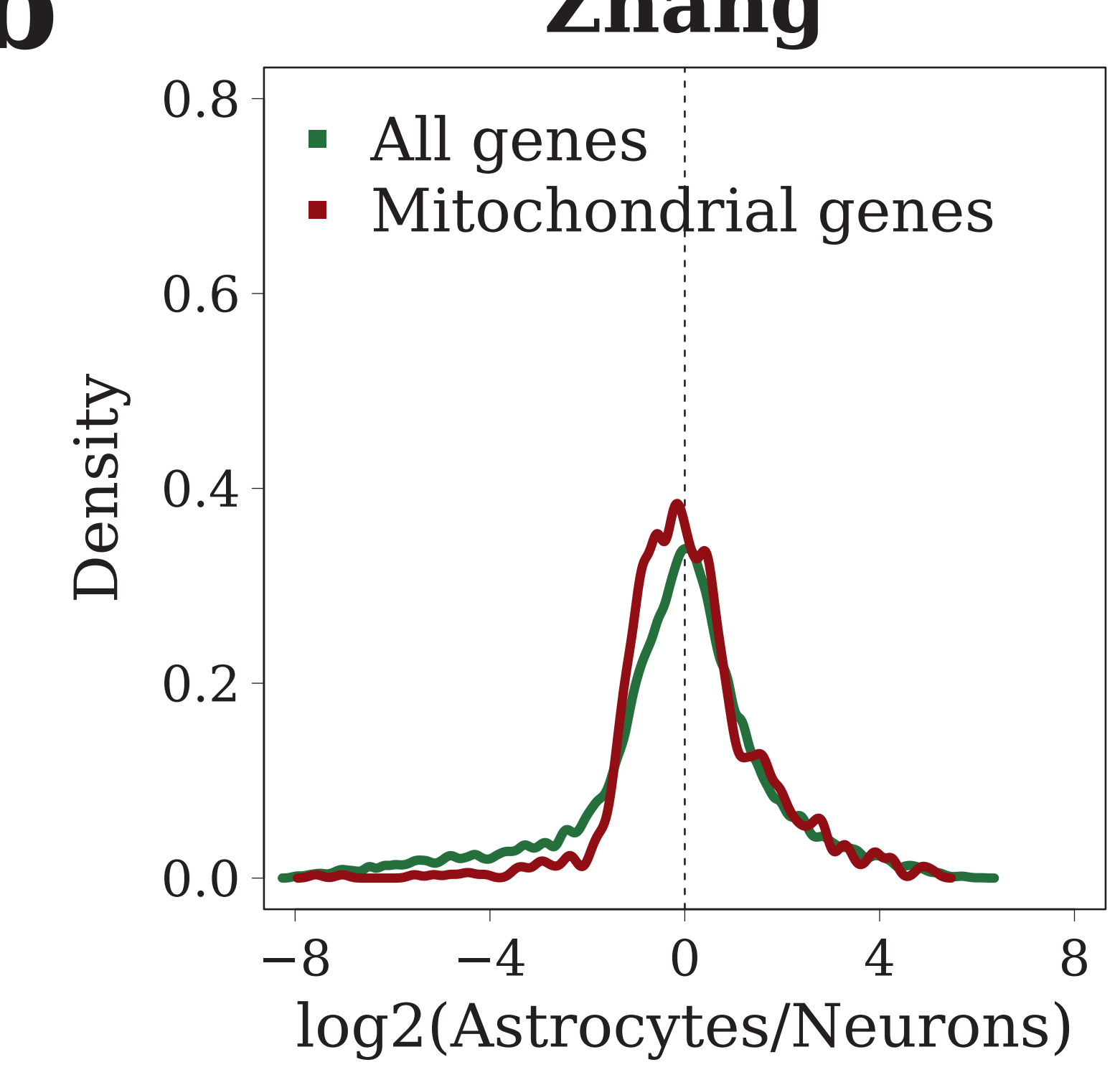

Doyle
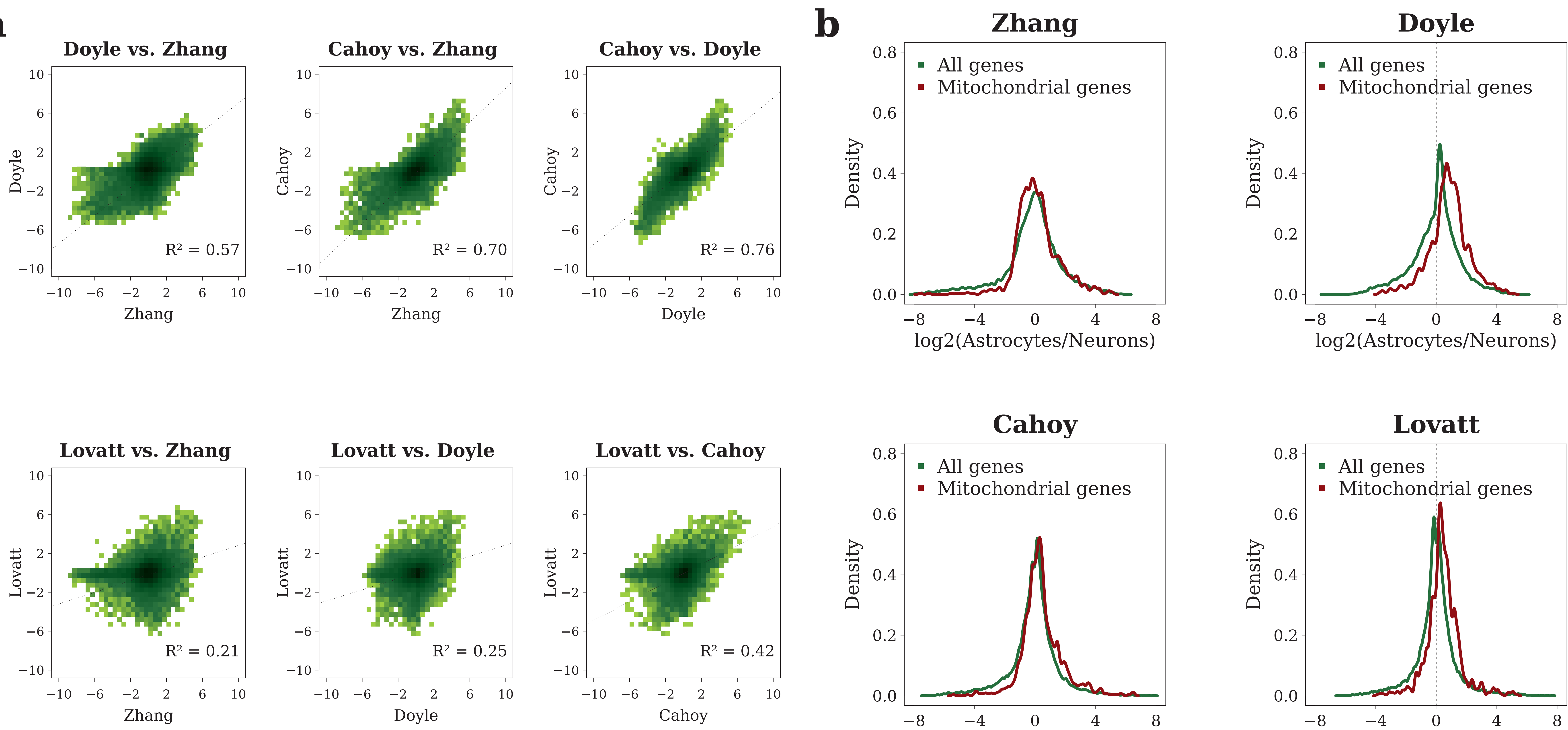

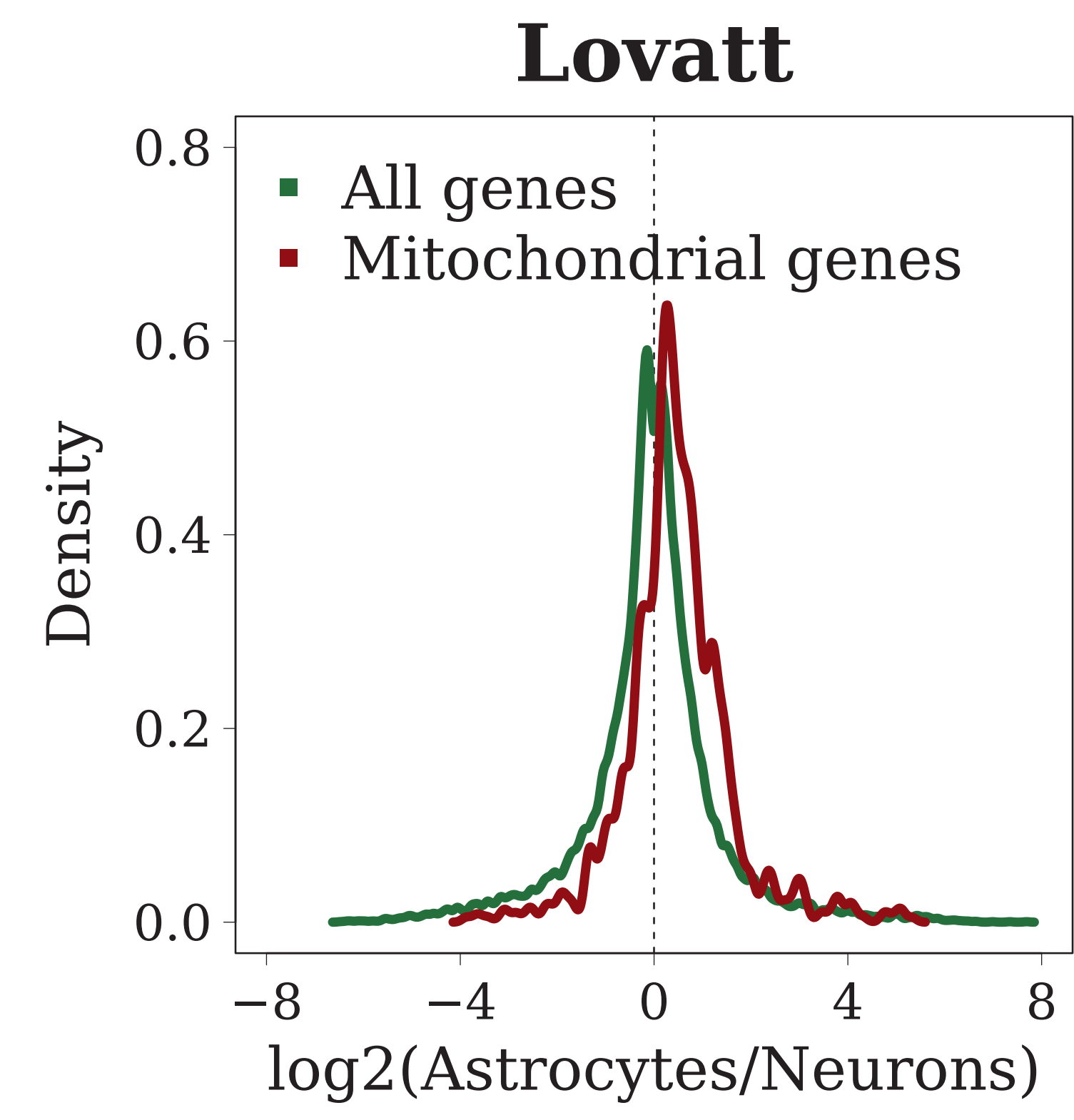


Amine, alcohol and aldehyde metabolism Cell death

T Cellular energy homeostasis

Carbohydrate catabolism

- Pyruvate metabolism

Lipid catabolism

- Fatty acid beta oxidation

Lipid synthesis

- Fatty acid synthesis

Oxidative metabolism

- Anaplerosis and cataplerosis

- Oxidative phosphorylation

- TCA

Tembrane lipid metabolism and dynamics Membrane lipid synthesis

- Glycerophospholipid synthesis

Mitochondrial biogenesis and dynamics Mitochondrial morphology

- Mitochondrial fusion and fission

- Mitochondrial trafficking

- Neurotransmitter metabolism

T Nucleic acid metabolism

- Mitochondrial DNA replication

Mitochondrial DNA translation

- Nucleotide synthesis

Protein homeostasis

Protein catabolism

- Amino acid catabolism

Hydrolysis and metabolism of peptides and proteins Protein folding

Protein synthesis

Transport of proteins

Redox homeostasis

Oxidative stress

- Signaling

Transport across membranes

Small molecule transport

- Unknown function a

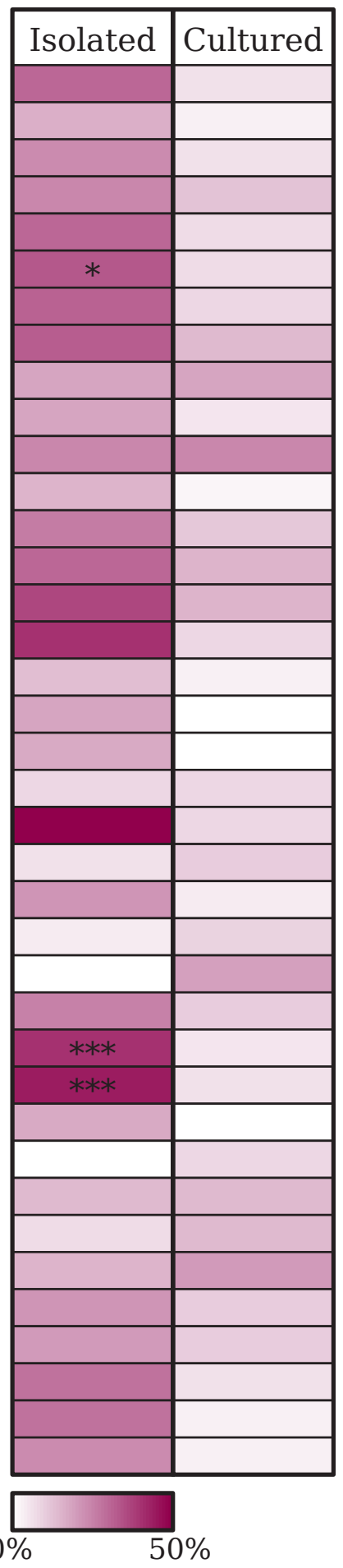

$\underset{+}{\text { Acyl-CoA }}$

b

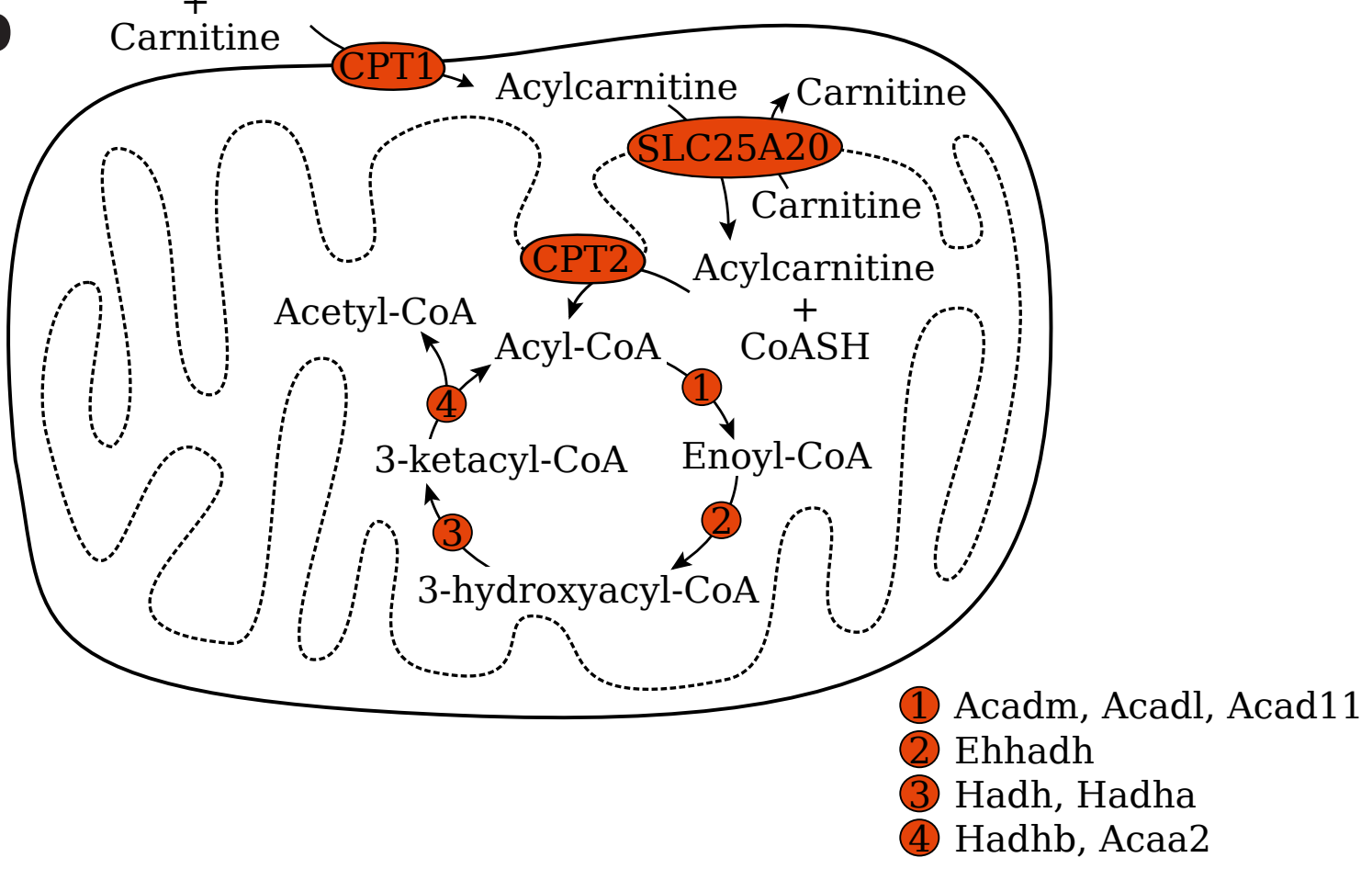

C

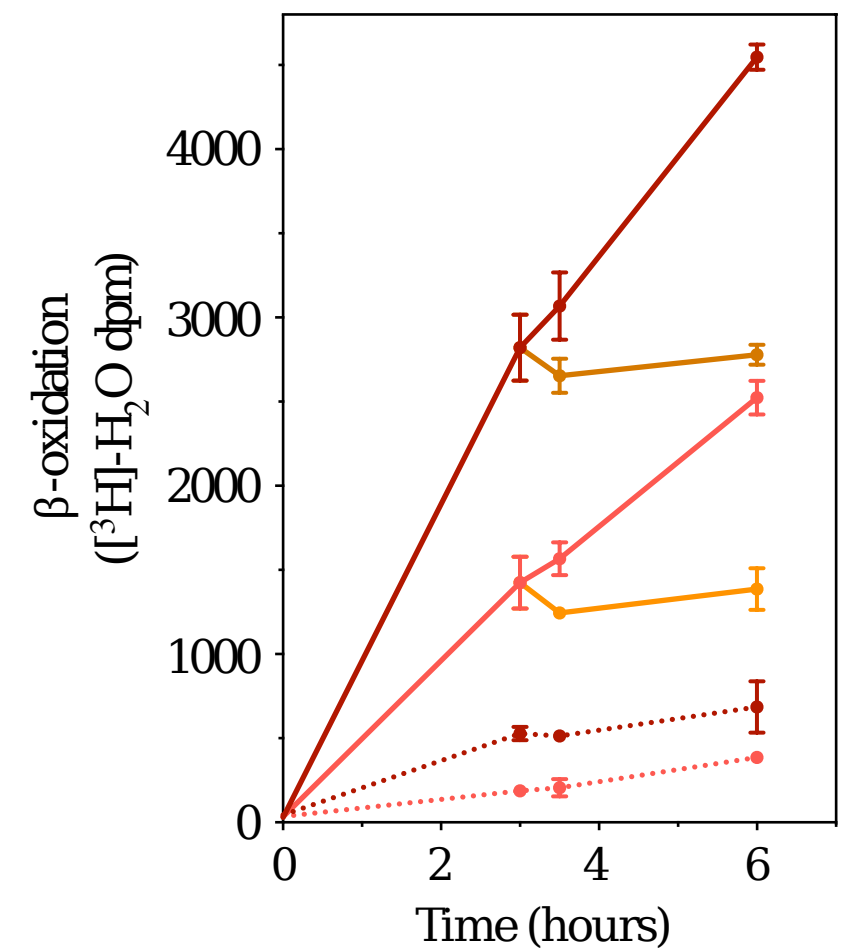

- No Glucose

- Glucose

$\rightarrow$ No Glucose + Glutamate

- Glucose + Glutamate

- No Glucose +ETX

- Glucose +ETX 
- Amine, alcohol and aldehyde metabolism Cell death

Cellular energy homeostasis

Carbohydrate catabolism

- Pyruvate metabolism

Lipid catabolism

Fatty acid beta oxidation

Lipid synthesis

- Fatty acid synthesis

Oxidative metabolism

- Anaplerosis and cataplerosis

- Oxidative phosphorylation

- TCA

Membrane lipid metabolism and dynamics

Membrane lipid synthesis

- Glycerophospholipid synthesis

Mitochondrial biogenesis and dynamics

Titochondrial morphology

- Mitochondrial fusion and fission

- Mitochondrial trafficking

- Neurotransmitter metabolism

Nucleic acid metabolism

Mitochondrial DNA replication

- Mitochondrial DNA translation

- Nucleotide synthesis

Protein homeostasis

Protein catabolism

- Amino acid catabolism

Hydrolysis and metabolism of peptides and proteins

Protein folding

Protein synthesis

Transport of protein

Tedox homeostasis

- Oxidative stress

- Signaling

Transport across membranes

- Small molecule transport

— Unknown function

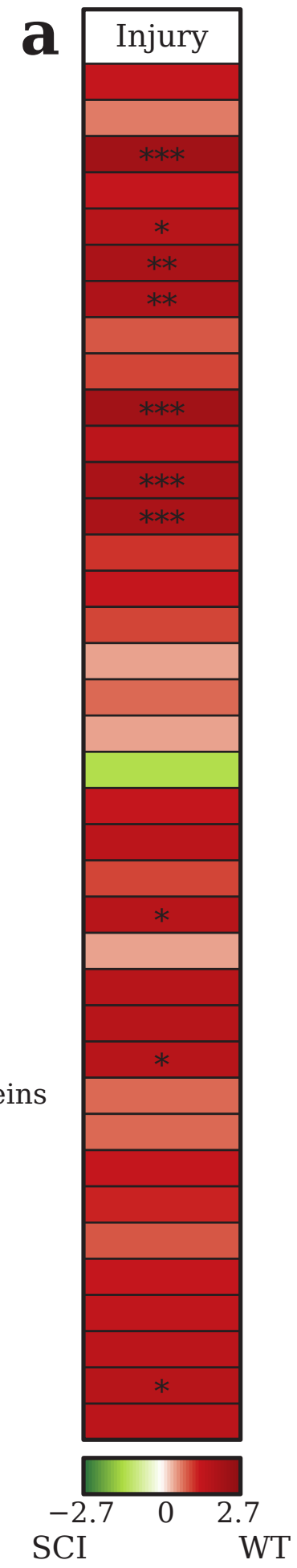

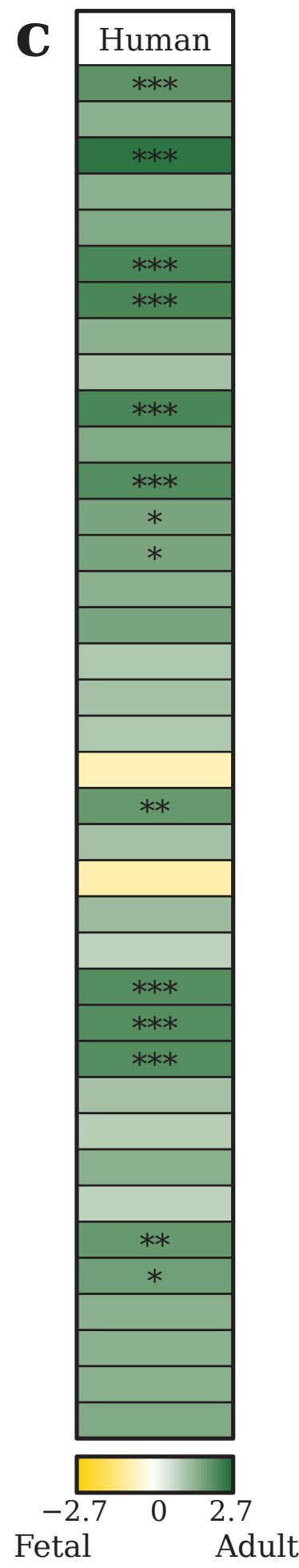

d
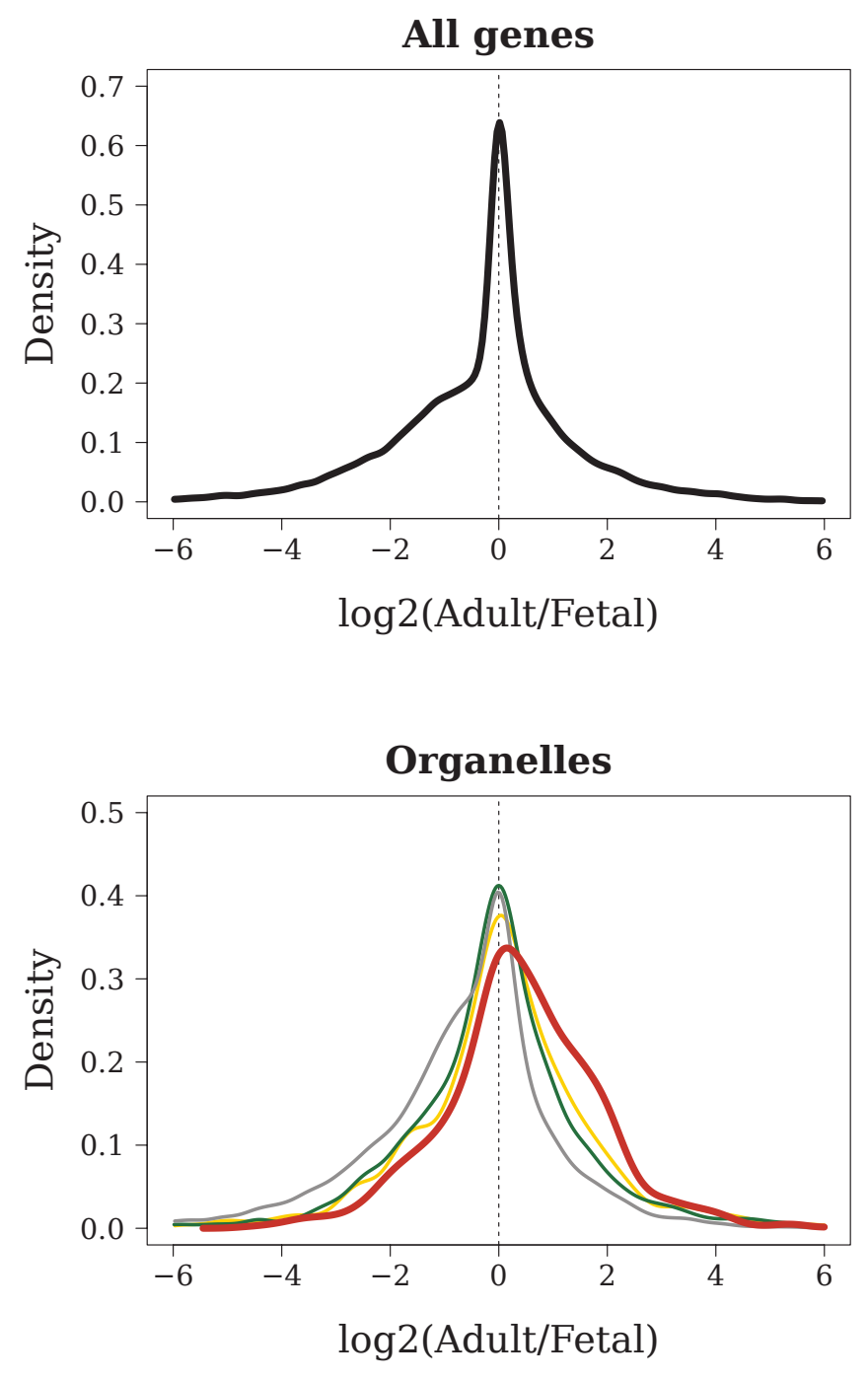

- Mitochondria

- Endoplasmic reticulum

- Golgi apparattus

- Nucleus 
- Amine, alcohol and aldehyde metabolism Cell death

Cellular energy homeostasis

Carbohydrate catabolism

Pyruvate metabolism

Lipid catabolism

- Fatty acid beta oxidation

Lipid synthesis

- Fatty acid synthesis

Oxidative metabolism

- Anaplerosis and cataplerosis

- Oxidative phosphorylation

TCA

Membrane lipid metabolism and dynamics

Membrane lipid synthesis

- Glycerophospholipid synthesis

Mitochondrial biogenesis and dynamics

Mitochondrial morphology

Mitochondrial fusion and fission

Mitochondrial trafficking

\section{- Neurotransmitter metabolism}

Nucleic acid metabolism

Mitochondrial DNA replication

- Mitochondrial DNA stability

- Mitochondrial DNA translation

- Nucleotide synthesis

Protein homeostasis

Protein catabolism

- Amino acid catabolism

Hydrolysis and metabolism of peptides and proteins Protein folding

Protein synthesis

- Transport of proteins

Redox homeostasis

- Oxidative stress

Signaling

Nuclear receptor signaling

Transport across membranes

Small molecule transport

- Unknown function

Anderson

SC Astrocytes

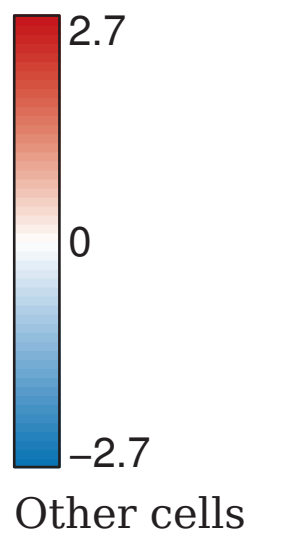

